\title{
The convenient setting for ultradifferentiable mappings of Beurling- and Roumieu-type defined by a weight matrix
}

\author{
Gerhard Schindl*
}

\begin{abstract}
We prove in a uniform way that all ultradifferentiable function classes $\mathcal{E}_{\{\mathcal{M}\}}$ of Roumieu-type and $\mathcal{E}_{(\mathcal{M})}$ of Beurling-type defined in terms of a weight matrix $\mathcal{M}$ admit a convenient setting if $\mathcal{M}$ satisfies some mild regularity conditions. For $\mathcal{C}$ denoting either $\mathcal{E}_{\{\mathcal{M}\}}$ or $\mathcal{E}_{(\mathcal{M})}$ the category $\mathcal{C}$ is cartesian closed, i.e. $\mathcal{C}(E \times F, G) \cong \mathcal{C}(E, \mathcal{C}(F, G))$ for $E, F, G$ convenient vector spaces. As special cases one obtains the classes $\mathcal{E}_{\{M\}}$ and $\mathcal{E}_{(M)}$ respectively $\mathcal{E}_{\{\omega\}}$ and $\mathcal{E}_{(\omega)}$ defined by a weight sequence $M$ respectively a weight function $\omega$.
\end{abstract}

\section{Introduction}

Spaces of ultradifferentiable functions are subclasses of smooth functions with certain growth conditions on all their derivatives. In the literature two different approaches are considered, either using a weight sequence $M=\left(M_{k}\right)_{k}$ or using a weight function $\omega$. For compact $K$ the set

$$
\left\{\frac{f^{(k)}(x)}{h^{k} M_{k}}: x \in K, k \in \mathbb{N}\right\} \quad \text { respectively } \quad\left\{\frac{f^{(k)}(x)}{\exp \left(1 / l \varphi_{\omega}^{*}(l k)\right)}: x \in K, k \in \mathbb{N}\right\}
$$

should be bounded, where the positive real number $h$ respectively $l$ is subject to either a universal or an existential quantifier and $\varphi_{\omega}^{*}$ denotes the Young-conjugate

${ }^{*}$ GS was supported by FWF-Project P 23028-N13 and FWF-Project P 26735-N25

Received by the editors in December 2014 - In revised form in February 2015.

Communicated by F. Bastin.

2010 Mathematics Subject Classification : 46E10, 46T05, 46T10.

Key words and phrases : Ultradifferentiable functions, convenient setting. 
of $\varphi_{\omega}=\omega \circ \exp$. In the case of a universal quantifier we call the class of Beurlingtype, denoted by $\mathcal{E}_{(M)}$ or $\mathcal{E}_{(\omega)}$, in the case of an existential quantifier we call the class of Roumieu-type, denoted by $\mathcal{E}_{\{M\}}$ or $\mathcal{E}_{\{\omega\}}$. We write $\mathcal{E}_{[\star]}$ if either $\mathcal{E}_{\{\star\}}$ or $\mathcal{E}_{(\star)}$ is considered.

That a class of mappings $\mathcal{C}$ admits a convenient setting means that one can extend the class to admissible infinite dimensional vector spaces $E, F, G$ such that $\mathcal{C}(E, F)$ is again admissible and the spaces $\mathcal{C}(E \times F, G)$ and $\mathcal{C}(E, \mathcal{C}(F, G))$ are canonically $\mathcal{C}$-diffeomorphic. This important property is called the exponential law.

We recall now some facts, see [4] or the appendix in [5] for a short overview. The class $\mathcal{E}$ of all smooth functions admits a convenient setting and for this approach one can test smoothness along $\mathcal{E}$-curves. The class $\mathcal{C}^{\omega}$ of all real-analytic mappings also admits a convenient setting. A mapping is $\mathcal{C}^{\omega}$ if and only if it is $\mathcal{E}$ and in addition it is weakly $\mathcal{C}^{\omega}$ along (weakly) $\mathcal{C}^{\omega}$-curves, i.e. curves whose compositions with any bounded linear functional are $\mathcal{C}^{\omega}$. It actually suffices to test along affine lines.

In [5], [7] and finally in [6] A. Kriegl, P.W. Michor and A. Rainer were able to develop the convenient setting for all reasonable classes $\mathcal{E}_{(M)}$ and $\mathcal{E}_{\{M\}}$. In the first step in [5] they introduced the convenient setting for $\mathcal{E}_{\{M\}}$ by testing with $\mathcal{E}_{\{M\}}$-curves for non-quasianalytic, strongly log-convex weight sequences $M$ of moderate growth. A function is $\mathcal{E}_{\{M\}}$ if and only if it is $\mathcal{E}_{\{M\}}$ along all $\mathcal{E}_{\{M\}}$-curves. It was shown that moderate growth is really necessary for the exponential law and non-quasianalyticity is needed for the existence of $\mathcal{E}_{\{M\}}$-partitions of unity.

Then, in [7], they succeeded to introduce the convenient setting for some quasianalytic classes $\mathcal{E}_{\{M\}}$. In this case $M$ has to satisfy again strong log-convexity, moderate growth and be such that $\mathcal{E}_{\{M\}}$ can be represented as the intersection of all larger non-quasianalytic classes $\mathcal{E}_{\{L\}}$ with strongly log-convex $L$. A mapping is $\mathcal{E}_{\{M\}}$ if and only if it is $\mathcal{E}_{\{L\}}$ along each $\mathcal{E}_{\{L\}}$-curve for each $L \geq M$ which is strongly log-convex and non-quasianalytic. A family of explicit examples $\mathcal{E}_{\{M\}}$ satisfying the requested assumptions was constructed, but the approach does not cover the real analytic case $\mathcal{C}^{\omega}$ and thus was not completely satisfactory.

Finally, in [6], it was shown that all classes $\mathcal{E}_{\{M\}}$ and $\mathcal{E}_{(M)}$ such that $M$ is strongly log-convex and has moderate growth admit a convenient setting, no matter if $M$ is quasianalytic or not. Instead of testing along curves the mappings are tested along Banach plots, i.e. mappings of the respective weak class defined in open subsets of Banach spaces. A smooth mapping between convenient vector spaces is $\mathcal{E}_{[M]}$ if it maps $\mathcal{E}_{[M]}$-Banach-plots to $\mathcal{E}_{[M]}$-Banach-plots.

The aim of this work is to generalize the results of [6] to classes $\mathcal{E}_{[\mathcal{M}]}$ defined by (one-parameter) weight matrices $\mathcal{M}:=\left\{M^{x}: x \in \mathbb{R}_{>0}\right\}$. In [9] the classes $\mathcal{E}_{[M]}$ and $\mathcal{E}_{[\omega]}$ were identified as particular cases of $\mathcal{E}_{[\mathcal{M}]}$. So using this new approach one is able to transfer results from one setting into the other one. Moreover one is able to prove results for $\mathcal{E}_{[M]}$ and $\mathcal{E}_{[\omega]}$ simultaneously and no longer two separate proofs are necessary. We have also shown that there are classes $\mathcal{E}_{[\mathcal{M}]}$ which cannot be described by a single $M$ or $\omega$, e.g. the class defined by the Gevrey-matrix $\mathcal{G}:=\left\{\left(p !^{!+1}\right)_{p \in \mathbb{N}}: s>0\right\}$. To transfer the proofs of [6] we will assume for $\mathcal{M}$ among mild basic properties the so-called generalized Fa-di-Bruno-property 
$\left(\mathcal{M}_{[\mathrm{FdB}]}\right)$ and the moderate growth condition $\left(\mathcal{M}_{[\mathrm{mg}]}\right)$.

After introducing the basic notation and definitions we recall the setting of Whitney jets between Banach spaces. We introduce classes of ultradifferentiable functions defined by weight matrices, first between Banach spaces and then between convenient vector spaces. This will be done in section 3 , In section 4 we are going to prove the most important and new tools in this work. We will develop projective descriptions for the classes $\mathcal{E}_{[\mathcal{M}]}$ in order to get rid of both existence quantifiers in the Roumieu-case (if $\mathcal{M}=\{M\}$ only one occurs). For this we have to use diagonal techniques and to introduce several families of sequences of positive real numbers to generalize the results of [6]. These projective representations are needed in section 5 for the proof of Theorem 5.9 to show that $\mathcal{E}_{[\mathcal{M}]}$ is a category and for cartesian closedness Theorem 6.2 in section 6 ,

Finally in section 7 we summarize some special cases. In 7.3 we revisit weight matrices as defined by Beaugendre in [1] and Schmets and Valdivia in [13]. Put $\mathcal{M}^{\Phi}:=\left\{\left(p ! m_{a p}^{\Phi}\right)_{p \in \mathbb{N}}: a>0\right\}$, where $\Phi:[0,+\infty) \rightarrow \mathbb{R}$ is a convex and increasing function with $\lim _{t \rightarrow \infty} \frac{\Phi(t)}{t}=+\infty, \Phi(0)=0$. In the literature only the Beurling-type-class was studied. We will see that the results in this work can also be applied to such classes.

Note that if $\mathcal{M}=\{M\}$ then the Fa-di-Bruno-property for $M$ is sufficient to show closedness under composition and is sufficient for the proofs in this work. But it is really weaker than strong log-convexity as assumed always in the previous papers and proofs of Kriegl, Michor, Rainer, see [9, 3.3.] for an explicit (counter)example. So our results are slightly more general than those of [6] even in the single weight sequence case. In Lemma 6.6 we will show that $\left(\mathcal{M}_{\{\mathrm{mg}\}}\right)$ is necessary for cartesian closedness of $\mathcal{E}_{\{\mathcal{M}\}}$ and in Example 6.5 we will point out that there exist weight matrices $\mathcal{M}$ such that no $M^{x} \in \mathcal{M}$ has moderate growth but nevertheless $\left(\mathcal{M}_{\{\mathrm{mg}\}}\right)$ is valid. In particular this holds if the matrix is associated to a weight function $\omega$ and such that $\mathcal{E}_{[\omega]}=\mathcal{E}_{[M]}$ does not hold, see [2] and [9].

This paper contains some of the main results of the authors PhD-Thesis, see [12]. The author thanks his advisor A. Kriegl, P.W. Michor and A. Rainer for the supervision and their helpful ideas.

\subsection{Basic notation}

We denote by $\mathcal{C}$ the class of all continuous, by $\mathcal{E}$ the class of smooth functions and $\mathcal{C}^{\omega}$ is the class of all real analytic functions. We will write $\mathbb{N}_{>0}=\{1,2, \ldots\}$, $\mathbb{N}=\mathbb{N}_{>0} \cup\{0\}$ and put $\mathbb{R}_{>0}:=\{x \in \mathbb{R}: x>0\}$. For $\alpha=\left(\alpha_{1}, \ldots, \alpha_{n}\right) \in \mathbb{N}^{n}$ we use the usual multi-index notation, write $\alpha !:=\alpha_{1} ! \ldots \alpha_{n} !,|\alpha|:=\alpha_{1}+\cdots+\alpha_{n}$ and for $x=\left(x_{1}, \ldots, x_{n}\right) \in \mathbb{R}^{n}$ we set $x^{\alpha}=x_{1}^{\alpha_{1}} \cdots x_{n}^{\alpha_{n}}$. We also put $\partial^{\alpha}=\partial_{1}^{\alpha_{1}} \cdots \partial_{n}^{\alpha_{n}}$ and denote by $f^{(k)}$ the $k$-th order Fréchet derivative of $f$. Iterated uni-directional derivatives are defined by $d_{v}^{k} f(x):=\left.\left(\frac{d}{d t}\right)^{k} f(x+t v)\right|_{t=0}$.

Let $E_{1}, \ldots, E_{k}$ and $F$ be topological vector spaces, then $L\left(E_{1}, \ldots, E_{k}, F\right)$ is the space of all bounded $k$-linear mappings $E_{1} \times \cdots \times E_{k} \rightarrow F$. If $E=E_{i}$ for $i=1, \ldots, k$, 
then we write $L^{k}(E, F) . L_{\text {sym }}^{k}(E, F)$ is the space of all symmetric $k$-linear bounded mappings $\underbrace{E \times \cdots \times E}_{k \text {-times }} \rightarrow F$, so $f^{(k)}: U \rightarrow L_{\text {sym }}^{k}(E, F)$. $E^{*}$ denotes the space of all continuous linear functionals on $E, E^{\prime}$ the space of all bounded linear functionals. If $B \subseteq E$ is closed absolutely convex bounded, then $E_{B}$ denotes the space generated by $B$ with the Minkowski-functional $\|\cdot\|_{B}$.

Let $E$ be a locally convex vector space, then the $c^{\infty}$-topology on $E$ is the final topology w.r.t. all smooth curves $c: \mathbb{R} \rightarrow E$. $E$ is called convenient if $E$ is $c^{\infty}$ complete which is equivalent for $E$ to be Mackey-complete and for $E_{B}$ to be a Banach space for every bounded absolutely convex subset $B$ of $E$. We refer to [4] or the appendix in [5] for more details and proofs.

Convention: Let $\star \in\{M, \omega, \mathcal{M}\}$, then write $\mathcal{E}_{[\star]}$ if either $\mathcal{E}_{\{\star\}}$ or $\mathcal{E}_{(\star)}$ is considered, but not mixing the cases if statements involve more than one $\mathcal{E}_{[\star]}$ symbol. The same notation will be used for the conditions, so write $\left(\mathcal{M}_{[\star]}\right)$ for either $\left(\mathcal{M}_{\{\star\}}\right)$ or $\left(\mathcal{M}_{(\star)}\right)$.

\section{Basic definitions}

\subsection{Weight sequences and classes of ultradifferentiable functions $\mathcal{E}_{[M]}$}

A weight sequence is an arbitrary sequence of positive real numbers $M=\left(M_{k}\right)_{k} \in \mathbb{R}_{>0}^{\mathbb{N}}$. We introduce also $m=\left(m_{k}\right)_{k}$ defined by $m_{k}:=\frac{M_{k}}{k !}$ and $\mu_{k}:=\frac{M_{k}}{M_{k-1}}, \mu_{0}:=1$. $M$ is called normalized if $1=M_{0} \leq M_{1}$ holds.

(1) $M$ is log-convex if

$$
\text { (lc) }: \Leftrightarrow \forall j \in \mathbb{N}: M_{j}^{2} \leq M_{j-1} M_{j+1} \text {. }
$$

$M$ is log-convex if and only if $\left(\mu_{k}\right)_{k}$ is increasing. If $M$ is log-convex and $M_{0}=1$, then

$$
\text { (alg) : } \Leftrightarrow \exists \geq 1 \forall j, k \in \mathbb{N}: M_{j} M_{k} \leq C^{j+k} M_{j+k}
$$

holds with $C=1$ and the mapping $j \mapsto\left(M_{j}\right)^{1 / j}$ is increasing, see e.g. [11, Lemma 2.0.4, Lemma 2.0.6].

$M$ is called strongly log-convex if

$$
\text { (slc) }: \Leftrightarrow \forall j \in \mathbb{N}: m_{j}^{2} \leq m_{j-1} m_{j+1} .
$$

This condition implies (lc) and was a basic assumptions for $M$ in [5], [7] and [6]. It guarantees all stability properties in [10, Theorems 5,6$]$ for the case $\mathcal{M}=\{M\}$, see also [9, Theorem 3.2.]. Related to this is the weaker condition

$$
(\mathrm{FdB}): \Leftrightarrow \exists D \geq 1 \forall k \in \mathbb{N}: m_{k}^{\circ} \leq D^{k} m_{k}
$$

which is called the Fa-di-Bruno-property, see [9, 3.3.]. For $m^{\circ}=\left(m_{k}^{\circ}\right)_{k}$ we have put

$$
m_{k}^{\circ}:=\max \left\{m_{j} m_{\alpha_{1}} \cdots m_{\alpha_{j}}: \alpha_{i} \in \mathbb{N}_{>0}, \sum_{i=1}^{j} \alpha_{i}=k\right\}, \quad m_{0}^{\circ}:=1 .
$$


Strongly log-convexity is also related to

$$
\text { (rai) : } \Leftrightarrow \exists C \geq 1 \forall 1 \leq j \leq k:\left(m_{j}\right)^{1 / j} \leq C\left(m_{k}\right)^{1 / k},
$$

see [9] and [10].

(2) $M$ has moderate growth if

$$
(\mathrm{mg}): \Leftrightarrow \exists C \geq 1 \forall j, k \in \mathbb{N}: M_{j+k} \leq C^{j+k} M_{j} M_{k} .
$$

This condition implies derivation closedness:

$$
\text { (dc) }: \Leftrightarrow \exists C \geq 1 \forall j \in \mathbb{N}: M_{j+1} \leq C^{j+1} M_{j} .
$$

In both conditions one can replace the sequence $M$ by $m$.

(3) For $M=\left(M_{p}\right)_{p}$ and $N=\left(N_{p}\right)_{p}$ we write $M \leq N$ if and only if $M_{p} \leq N_{p}$ for all $p \in \mathbb{N}$. Moreover we define

$$
M \preceq N: \Leftrightarrow \exists C_{1}, C_{2} \geq 1 \forall j \in \mathbb{N}: M_{j} \leq C_{2} C_{1}^{j} N_{j} \Longleftrightarrow \sup _{p \in \mathbb{N}_{>0}}\left(\frac{M_{p}}{N_{p}}\right)^{1 / p}<+\infty
$$

and we call the sequences equivalent if

$$
M \approx N: \Leftrightarrow M \preceq N \text { and } N \preceq M .
$$

We will write

$$
M \triangleleft N: \Leftrightarrow \forall h>0 \exists C_{h} \geq 1 \forall j \in \mathbb{N}: M_{j} \leq C_{h} h^{j} N_{j} \Longleftrightarrow \lim _{p \rightarrow \infty}\left(\frac{M_{p}}{N_{p}}\right)^{1 / p}=0 .
$$

For convenience we introduce the following set:

$$
\mathcal{L C}:=\left\{M \in \mathbb{R}_{>0}^{\mathbb{N}}: M \text { is normalized, log-convex, } \lim _{k \rightarrow \infty}\left(M_{k}\right)^{1 / k}=+\infty\right\} .
$$

Let $r, s \in \mathbb{N}_{>0}$ and $U \subseteq \mathbb{R}^{r}$ be non-empty open. We introduce the ultradifferentiable class of Roumieu-type by

$$
\mathcal{E}_{\{M\}}\left(U, \mathbb{R}^{s}\right):=\left\{f \in \mathcal{E}\left(U, \mathbb{R}^{s}\right): \forall K \subseteq U \text { compact } \exists h>0:\|f\|_{M, K, h}<+\infty\right\},
$$

and the class of Beurling-type by

$$
\mathcal{E}_{(M)}\left(U, \mathbb{R}^{s}\right):=\left\{f \in \mathcal{E}\left(U, \mathbb{R}^{s}\right): \forall K \subseteq U \text { compact } \forall h>0:\|f\|_{M, K, h}<+\infty\right\},
$$

where we have put

$$
\|f\|_{M, K, h}:=\sup _{k \in \mathbb{N}, x \in K} \frac{\left\|f^{(k)}(x)\right\|_{L^{k}\left(\mathbb{R}^{r}, \mathbb{R}^{s}\right)}}{h^{k} M_{k}} .
$$

For compact sets $K$ with smooth boundary

$$
\mathcal{E}_{M, h}\left(K, \mathbb{R}^{s}\right):=\left\{f \in \mathcal{E}\left(K, \mathbb{R}^{s}\right):\|f\|_{M, K, h}<+\infty\right\}
$$


is a Banach space and we have the topological vector space representations

$$
\mathcal{E}_{\{M\}}\left(U, \mathbb{R}^{S}\right):=\lim _{K \subseteq U} \underset{h>0}{\lim _{h}} \mathcal{E}_{M, h}\left(K, \mathbb{R}^{S}\right)=\lim _{K \subseteq U} \mathcal{E}_{\{M\}}\left(K, \mathbb{R}^{S}\right)
$$

and

$$
\mathcal{E}_{(M)}\left(U, \mathbb{R}^{S}\right):=\lim _{K \subseteq U} \lim _{h>0} \mathcal{E}_{M, h}\left(K, \mathbb{R}^{S}\right)=\lim _{K \subseteq U} \mathcal{E}_{(M)}\left(K, \mathbb{R}^{S}\right)
$$

We recall some facts for log-convex $M$ :

(i) Put $\mathcal{E}_{\{M\}}^{\text {global }}\left(U, \mathbb{R}^{s}\right):=\left\{f \in \mathcal{E}\left(U, \mathbb{R}^{s}\right): \exists h>0\|f\|_{M, U, h}<+\infty\right\}$. There exist characteristic functions

$$
(\text { chf }): \Leftrightarrow \exists \theta_{M} \in \mathcal{E}_{\{M\}}^{\text {global }}(\mathbb{R}, \mathbb{R}): \forall j \in \mathbb{N}:\left|\theta_{M}^{(j)}(0)\right| \geq M_{j}
$$

and $\tilde{\theta}_{M} \in \mathcal{E}_{\{M\}}^{\text {global }}(\mathbb{R}, \mathbb{C})$ with

$$
\forall j \in \mathbb{N}: \tilde{\theta}_{M}^{(j)}(0)=(\sqrt{-1})^{j} s_{j}, \quad s_{j}:=\sum_{k=0}^{\infty} M_{k}\left(2 \mu_{k}\right)^{j-k} \geq M_{j},
$$

hence $\left|\tilde{\theta}_{M}^{(j)}(0)\right| \geq M_{j}$ for all $j \in \mathbb{N}$, see [9, Lemma 2.9.] and [14, Theorem 1]. Note that the Beurling-class $\mathcal{E}_{(M)}^{\text {global }}(\mathbb{R}, \mathbb{R})$ cannot contain such $\theta_{M}$, see [11, Proposition 3.1.2.].

(ii) If $N$ is arbitrary, then $M \preceq N \Longleftrightarrow \mathcal{E}_{\{M\}} \subseteq \mathcal{E}_{\{N\}}$ and $M \triangleleft \mathbb{N} \Longleftrightarrow \mathcal{E}_{\{M\}} \subseteq$ $\mathcal{E}_{(N)}$. If $M \in \mathcal{L C}$, then $M \preceq N \Longleftrightarrow \mathcal{E}_{[M]} \subseteq \mathcal{E}_{[N]}$.

(iii) Both classes $\mathcal{E}_{\{M\}}$ and $\mathcal{E}_{(M)}$ are closed under pointwise multiplication, see e.g. [11, Proposition 2.0.8].

\subsection{Classes of ultra-differentiable functions defined by one parameter weight matrices and basic definitions}

Definition 2.3. Let $(\Lambda, \leq)$ be a partially ordered set which is both up-and downward directed, $\Lambda=\mathbb{R}_{>0}$ will be the most important example. A weight matrix $\mathcal{M}$ associated to $\Lambda$ is a family of weight sequences $\mathcal{M}:=\left\{M^{x} \in \mathbb{R}_{>0}^{\mathbb{N}}: x \in \Lambda\right\}$ such that

$$
(\mathcal{M}): \Leftrightarrow \forall x \in \Lambda: M^{x} \text { is normalized, increasing, } M^{x} \leq M^{y} \text { for } x \leq y \text {. }
$$

We call $\mathcal{M}$ standard log-convex, if

$$
\left(\mathcal{M}_{\mathrm{sc}}\right): \Leftrightarrow(\mathcal{M}) \text { and } \forall x \in \Lambda: M^{x} \in \mathcal{L C} \text {. }
$$

Also $m_{k}^{x}:=\frac{M_{k}^{x}}{k !}$ and $\mu_{k}^{x}:=\frac{M_{k}^{x}}{M_{k-1}^{x}}, \mu_{0}^{x}:=1$, will be used. 
We introduce ultradifferentiable classes of Roumieu- and Beurling-type defined by $\mathcal{M}$ as follows (see also [9, 4.2.]):

Let $r, s \in \mathbb{N}_{>0}$, let $U \subseteq \mathbb{R}^{r}$ be non-empty and open. For all $K \subseteq U$ compact we put

$$
\mathcal{E}_{\{\mathcal{M}\}}\left(K, \mathbb{R}^{s}\right):=\bigcup_{x \in \Lambda} \mathcal{E}_{\left\{M^{x}\right\}}\left(K, \mathbb{R}^{s}\right) \quad \mathcal{E}_{\{\mathcal{M}\}}\left(U, \mathbb{R}^{s}\right):=\bigcap_{K \subseteq U} \bigcup_{x \in \Lambda} \mathcal{E}_{\left\{M^{x}\right\}}\left(K, \mathbb{R}^{s}\right)
$$

and

$$
\mathcal{E}_{(\mathcal{M})}\left(K, \mathbb{R}^{s}\right):=\bigcap_{x \in \Lambda} \mathcal{E}_{\left(M^{x}\right)}\left(K, \mathbb{R}^{s}\right) \quad \mathcal{E}_{(\mathcal{M})}\left(U, \mathbb{R}^{s}\right):=\bigcap_{x \in \Lambda} \mathcal{E}_{\left(M^{x}\right)}\left(U, \mathbb{R}^{s}\right) .
$$

For a compact set $K \subseteq \mathbb{R}^{r}$ (with smooth boundary) we have

$$
\mathcal{E}_{\{\mathcal{M}\}}\left(K, \mathbb{R}^{s}\right):=\underset{x \in \Lambda}{\lim } \underset{h>0}{\lim } \mathcal{E}_{M^{x}, h}\left(K, \mathbb{R}^{s}\right),
$$

and so for $U \subseteq \mathbb{R}^{r}$ non-empty open

$$
\mathcal{E}_{\{\mathcal{M}\}}\left(U, \mathbb{R}^{s}\right):=\lim _{\overleftarrow{K \subseteq U}} \lim _{x \in \Lambda} \lim _{h>0} \mathcal{E}_{M^{x}, h}\left(K, \mathbb{R}^{s}\right),
$$

and for the Beurling-case we get

$$
\mathcal{E}_{(\mathcal{M})}\left(U, \mathbb{R}^{s}\right):=\varliminf_{K \subseteq U} \lim _{x \in \Lambda} \varliminf_{h>0} \mathcal{E}_{M^{x}, h}\left(K, \mathbb{R}^{s}\right)
$$

Instead of compact sets $K$ with smooth boundary one can also consider open $K \subseteq U$ with $\bar{K}$ compact in $U$, or one can work with Whitney jets on compact $K$. If $\Lambda=\mathbb{R}_{>0}$ we can assume that all occurring limits are countable and so

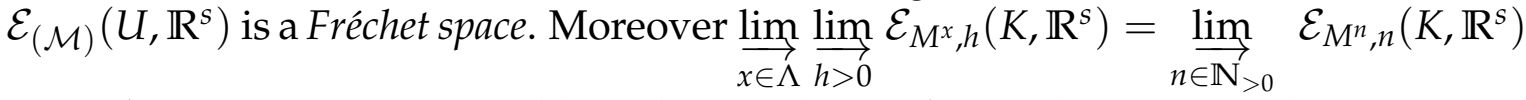
is a Silva space, i.e. a countable inductive limit of Banach spaces with compact connecting mappings. For more details concerning the locally convex topology on these spaces we refer to [9, 4.2.-4.4.].

\subsection{Conditions for a weight matrix $\mathcal{M}=\left\{M^{x}: x \in \Lambda\right\}$}

We are going to introduce now some conditions on $\mathcal{M}$ which will be needed frequently, see also [9, 4.1.].

Roumieu-type-conditions

$$
\begin{aligned}
& \left(\mathcal{M}_{\{\mathrm{dc}\}}\right) \forall x \in \Lambda \exists C>0 \exists y \in \Lambda \forall j \in \mathbb{N}: M_{j+1}^{x} \leq C^{j+1} M_{j}^{y} \\
& \left(\mathcal{M}_{\{\mathrm{mg}\}}\right) \forall x \in \Lambda \exists C>0 \exists y_{1}, y_{2} \in \Lambda \forall j, k \in \mathbb{N}: M_{j+k}^{x} \leq C^{j+k} M_{j}^{y_{1}} M_{k}^{y_{2}} \\
& \left(\mathcal{M}_{\{\mathrm{alg}\}}\right) \forall x_{1}, x_{2} \in \Lambda \exists C>0 \exists y \in \Lambda \forall j, k \in \mathbb{N}: M_{j}^{x_{1}} M_{k}^{x_{2}} \leq C^{j+k} M_{j+k}^{y} \\
& \left(\mathcal{M}_{\{\mathrm{L}\}}\right) \forall C>0 \forall x \in \Lambda \exists D>0 \exists y \in \Lambda \forall k \in \mathbb{N}: C^{k} M_{k}^{x} \leq D M_{k}^{y}
\end{aligned}
$$




$$
\begin{aligned}
& \left(\mathcal{M}_{\{\text {strict\} }}\right) \forall x \in \Lambda \exists y \in \Lambda: \sup _{k \in \mathbb{N}_{>0}}\left(\frac{M_{k}^{y}}{M_{k}^{x}}\right)^{1 / k}=+\infty \\
& \left(\mathcal{M}_{\{\text {FdB }\}}\right) \forall x \in \Lambda \exists y \in \Lambda:\left(m^{x}\right)^{q} \preceq m^{y} \\
& \left(\mathcal{M}_{\{\text {rai }\}}\right) \forall x \in \Lambda \exists y \in \Lambda \exists H>0:\left(m_{q}^{x}\right)^{1 / q} \leq H\left(m_{p}^{y}\right)^{1 / p}, 1 \leq q \leq p \\
& \quad \text { Beurling-type-conditions } \\
& \left(\mathcal{M}_{(\mathrm{dc})}\right) \forall x \in \Lambda \exists C>0 \exists y \in \Lambda \forall j \in \mathbb{N}: M_{j+1}^{y} \leq C^{j+1} M_{j}^{x} \\
& \left(\mathcal{M}_{(\mathrm{mg})}\right) \forall x_{1}, x_{2} \in \Lambda \exists C>0 \exists y \in \Lambda \forall j, k \in \mathbb{N}: M_{j+k}^{y} \leq C^{j+k} M_{j}^{x_{1}} M_{k}^{x_{2}} \\
& \left(\mathcal{M}_{(\mathrm{alg})}\right) \forall x \in \Lambda \exists C>0 \exists y_{1}, y_{2} \in \Lambda \forall j, k \in \mathbb{N}: M_{j}^{y_{1}} M_{k}^{y_{2}} \leq C^{j+k} M_{j+k}^{x} \\
& \left(\mathcal{M}_{(\mathrm{L})}\right) \forall C>0 \forall x \in \Lambda \exists D>0 \exists y \in \Lambda \forall k \in \mathbb{N}: C^{k} M_{k}^{y} \leq D M_{k}^{x} \\
& \left(\mathcal{M}_{(\text {strict })}\right) \forall x \in \Lambda \exists y \in \Lambda: \sup _{k \in \mathbb{N}_{>0}}\left(\frac{M_{k}^{x}}{M_{k}^{y}}\right)^{1 / k}=+\infty \\
& \left(\mathcal{M}_{(\mathrm{FdB})}\right) \forall x \in \Lambda \exists y \in \Lambda:\left(m^{y}\right)^{q} \preceq m^{x} \\
& \left(\mathcal{M}_{(\text {rai })}\right) \forall x \in \Lambda \exists y \in \Lambda \exists H>0:\left(m_{q}^{y}\right)^{1 / q} \leq H\left(m_{p}^{x}\right)^{1 / p}, 1 \leq q \leq p
\end{aligned}
$$

\subsection{Inclusion relations of weight matrices}

Let two matrices $\mathcal{M}=\left\{M^{x}: x \in \Lambda\right\}$ and $\mathcal{N}=\left\{N^{x}: x \in \Lambda^{\prime}\right\}$ be given, then we write

$$
\begin{aligned}
& \mathcal{M}\{\preceq\} \mathcal{N}: \Leftrightarrow \forall x \in \Lambda \exists y \in \Lambda^{\prime}: M^{x} \preceq \mathbb{N}^{y} \\
& \mathcal{M}(\preceq) \mathcal{N}: \Leftrightarrow \forall y \in \Lambda^{\prime} \exists x \in \Lambda: M^{x} \preceq N^{y},
\end{aligned}
$$

and

$$
\mathcal{M}\{\approx\} \mathcal{N}: \Leftrightarrow \mathcal{M}\{\preceq\} \mathcal{N} \text { and } \mathcal{N}\{\preceq\} \mathcal{M}
$$

respectively

$$
\mathcal{M}(\approx) \mathcal{N}: \Leftrightarrow \mathcal{M}(\preceq) \mathcal{N} \text { and } \mathcal{N}(\preceq) M .
$$

By definition $\mathcal{M}[\preceq] \mathcal{N}$ implies $\mathcal{E}_{[\mathcal{M}]} \subseteq \mathcal{E}_{[\mathcal{N}]}$. Moreover write

$$
\mathcal{M} \triangleleft \mathcal{N}: \Leftrightarrow \forall x \in \Lambda \forall y \in \Lambda^{\prime}: M^{x}\left[\mathbb{N}^{y},\right.
$$

so $\mathcal{M} \triangleleft \mathcal{N}$ implies $\mathcal{E}_{\{\mathcal{M}\}} \subseteq \mathcal{E}_{(\mathcal{N})}$. In [9, Proposition 4.6.] the above relations are characterized for $\left(\mathcal{M}_{\mathrm{sc}}\right)$-matrices with $\Lambda=\Lambda^{\prime}=\mathbb{R}_{>0}$. In this context we introduce

$\left(\mathcal{M}_{\left\{\mathcal{C}^{\omega}\right\}}\right) \exists x \in \Lambda: \liminf _{k \rightarrow \infty}\left(m_{k}^{x}\right)^{1 / k}>0$, $\left(\mathcal{M}_{\mathcal{H}}\right) \quad \forall x \in \Lambda: \liminf _{k \rightarrow \infty}\left(m_{k}^{x}\right)^{1 / k}>0$, $\left(\mathcal{M}_{\left(\mathcal{C}^{\omega}\right)}\right) \forall x \in \Lambda: \lim _{k \rightarrow \infty}\left(m_{k}^{x}\right)^{1 / k}=+\infty$.

If $\left(\mathcal{M}_{\left\{\mathcal{C}^{\omega}\right\}}\right)$ holds, then $\mathcal{C}^{\omega} \subseteq \mathcal{E}_{\{\mathcal{M}\}}$, if $\left.\left(\mathcal{M}_{\left(\mathcal{C}^{\omega}\right)}\right)\right]$ then $\mathcal{C}^{\omega} \subseteq \mathcal{E}_{(\mathcal{M})}$. Finally if $\left(\mathcal{M}_{\mathcal{H}}\right)$, then the restrictions of entire functions are contained in $\mathcal{E}_{(\mathcal{M})}$, see [9, Proposition 4.6.]. 


\section{Conventions:}

(i) If $\Lambda=\mathbb{R}_{>0}$ or $\Lambda=\mathbb{N}_{>0}$, then these sets are always regarded with its natural order $\leq$.

(ii) We will call $\mathcal{M}$ constant if $\mathcal{M}=\{M\}$ or more generally if $M^{x} \approx M^{y}$ for all $x, y \in \Lambda$ and which violates both $\left(\mathcal{M}_{\text {\{strict }\}}\right)$ and $\left(\mathcal{M}_{\text {(strict) }}\right)$. Otherwise it will be called non-constant.

\subsection{Weight functions and classes of ultradifferentiable functions $\mathcal{E}_{[\omega]}$}

A function $\omega:[0, \infty) \rightarrow[0, \infty)$ (sometimes $\omega$ is extended to $\mathbb{C}$ by $\omega(x):=\omega(|x|)$ ) is called a weight function if

$\left(\omega_{0}\right) \omega$ is continuous, on $[0, \infty)$ increasing, $\omega(x)=0$ for $x \in[0,1]$ (w.l.o.g.) and $\lim _{x \rightarrow \infty} \omega(x)=+\infty$.

Moreover we consider the following conditions:

$\left(\omega_{1}\right) \omega(2 t)=O(\omega(t))$ as $t \rightarrow+\infty$.

$\left(\omega_{2}\right) \omega(t)=O(t)$ as $t \rightarrow \infty$.

$\left(\omega_{3}\right) \log (t)=o(\omega(t))$ as $t \rightarrow+\infty\left(\Leftrightarrow \lim _{t \rightarrow+\infty} \frac{t}{\varphi_{\omega}(t)}=0\right)$.

$\left(\omega_{4}\right) \varphi_{\omega}: t \mapsto \omega\left(e^{t}\right)$ is a convex function on $\mathbb{R}$.

$\left(\omega_{5}\right) \omega(t)=o(t)$ as $t \rightarrow+\infty$.

$\left(\omega_{6}\right) \exists H \geq 1 \forall t \geq 0: 2 \omega(t) \leq \omega(H t)+H$.

$\left(\omega_{1^{\prime}}\right) \exists D>0: \exists t_{0}>0: \forall \lambda \geq 1: \forall t \geq t_{0}: \omega(\lambda t) \leq D \lambda \omega(t)$.

An interesting example is $\omega_{s}(t):=\max \left\{0, \log (t)^{s}\right\}, s>1$, which satisfies all listed properties except $\left(\omega_{6}\right)$. For convenience we define the sets

$$
\begin{gathered}
\mathcal{W}_{0}:=\left\{\omega:[0, \infty) \rightarrow[0, \infty): \omega \text { has }\left(\omega_{0}\right),\left(\omega_{3}\right),\left(\omega_{4}\right)\right\}, \\
\mathcal{W}:=\left\{\omega \in \mathcal{W}_{0}: \omega \text { has }\left(\omega_{1}\right)\right\} .
\end{gathered}
$$

For $\omega \in \mathcal{W}_{0}$ we define the Legendre-Fenchel-Young-conjugate $\varphi_{\omega}^{*}$ by

$$
\varphi_{\omega}^{*}(x):=\sup \left\{x y-\varphi_{\omega}(y): y \geq 0\right\}, \quad x \geq 0 .
$$

It is a convex increasing function, $\varphi_{\omega}^{*}(0)=0, \varphi_{\omega}^{* *}=\varphi_{\omega}, \lim _{x \rightarrow \infty} \frac{x}{\varphi_{\omega}^{*}(x)}=0$ and finally $x \mapsto \frac{\varphi_{\omega}(x)}{x}$ and $x \mapsto \frac{\varphi_{\omega}^{*}(x)}{x}$ are increasing on $[0,+\infty)$, see e.g. [3, Remark 1.3., Lemma 1.5.].

For $\sigma, \tau \in \mathcal{W}$ we write

$$
\sigma \preceq \tau: \Leftrightarrow \tau(t)=O(\sigma(t)), \text { as } t \rightarrow+\infty
$$


and call them equivalent if

$$
\sigma \sim \tau: \Leftrightarrow \sigma \preceq \tau \text { and } \tau \preceq \sigma \text {. }
$$

Let $r, s \in \mathbb{N}_{>0}, U \subseteq \mathbb{R}^{r}$ be a non-empty open set and $\omega \in \mathcal{W}_{0}$, The Roumieu-type space is defined by

$$
\mathcal{E}_{\{\omega\}}\left(U, \mathbb{R}^{s}\right):=\left\{f \in \mathcal{E}\left(U, \mathbb{R}^{s}\right): \forall K \subseteq U \text { compact } \exists l>0:\|f\|_{\omega, K, l}<+\infty\right\}
$$

and the Beurling-type space by

$$
\mathcal{E}_{(\omega)}\left(U, \mathbb{R}^{s}\right):=\left\{f \in \mathcal{E}\left(U, \mathbb{R}^{s}\right): \forall K \subseteq U \text { compact } \forall l>0:\|f\|_{\omega, K, l}<+\infty\right\},
$$

where we have put

$$
\|f\|_{\omega, K, l}:=\sup _{k \in \mathbb{N}, x \in K} \frac{\left\|f^{(k)}(x)\right\|_{L^{k}\left(\mathbb{R}^{r}, \mathbb{R}^{s}\right)}}{\exp \left(\frac{1}{\tau} \varphi_{\omega}^{*}(l k)\right)}
$$

and $f^{(k)}(x)$ denotes the $k$-th order Fréchet derivative at $x$. For compact sets $K$ with smooth boundary

$$
\mathcal{E}_{\omega, l}\left(K, \mathbb{R}^{s}\right):=\left\{f \in \mathcal{E}\left(K, \mathbb{R}^{s}\right):\|f\|_{\omega, K, l}<+\infty\right\}
$$

is a Banach space and we have the topological vector space representations

$$
\mathcal{E}_{\{\omega\}}\left(U, \mathbb{R}^{s}\right):=\lim _{\overleftarrow{K \subseteq U}} \lim _{l>0} \mathcal{E}_{\omega, l}\left(K, \mathbb{R}^{s}\right)=\lim _{\overleftarrow{K \subseteq U}} \mathcal{E}_{\{\omega\}}\left(K, \mathbb{R}^{s}\right)
$$

and

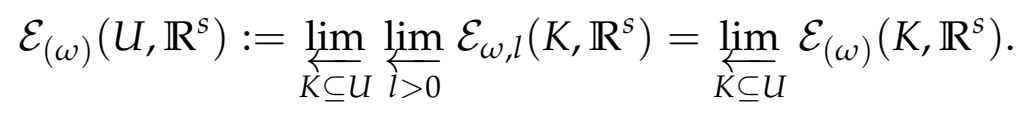

A new idea introduced in [9, Chapter 5] was the following:

(i) To each $\omega \in \mathcal{W}$ we can associate a $\left(\mathcal{M}_{\mathrm{sc}}\right)$ weight matrix $\Omega=\left\{\Omega^{l}=\left(\Omega_{j}^{l}\right)_{j \in \mathbb{N}}: l>0\right\}$ by

$$
\Omega_{j}^{l}:=\exp \left(\frac{1}{l} \varphi_{\omega}^{*}(l j)\right) .
$$

(ii) $\Omega$ has always $\left(\mathcal{M}_{\{\mathrm{mg}\}}\right)$ and $\left(\mathcal{M}_{(\mathrm{mg})}\right),\left(\mathcal{M}_{\{\mathrm{L}\}}\right)$ and $\left(\mathcal{M}_{(\mathrm{L})}\right)$, If $\omega$ is subadditive, then $\left(\mathcal{M}_{\{\mathrm{FdB}\}}\right)$ and $\left(\mathcal{M}_{(\mathrm{FdB})}\right)$ hold, see [9, Lemma 6.1.]. Equivalent weight functions $\omega$ yield equivalent weight matrices w.r.t. both $(\approx)$ and $\{\approx\}$.

(iii) $\mathcal{E}_{[\Omega]}=\mathcal{E}_{[\omega]}$ holds as locally convex vector spaces, so defining classes of ultradifferentiable functions by weight matrices as in (2.5) and (2.6) is a common generalization of defining them by using a single weight sequence $M$, i.e. a constant weight matrix, or a weight function $\omega \in \mathcal{W}$. But one is also able to describe classes which cannot be described neither by a weight function nor by a weight sequence, e.g. the class defined by the Gevrey-matrix $\mathcal{G}:=\left\{\left(p !^{s+1}\right)_{p \in \mathbb{N}}: s>0\right\}$, see [9, 5.19.]. 


\section{Basic definitions for the convenient setting}

\subsection{Whitney jets on Banach spaces}

We recall the notation of [6, Chapter 3]. Let $E, F$ be Banach spaces, $K \subseteq E$ compact and $U \subseteq E$ open. Let $f \in \mathcal{E}(U, F)$, then we introduce the jet mapping $j^{\infty}: \mathcal{E}(U, F) \rightarrow J^{\infty}(U, F):=\prod_{k \in \mathbb{N}} \mathcal{C}\left(U, L_{\text {sym }}^{k}(E, F)\right)$ defined by $f \mapsto j^{\infty}(f)=$ $\left(f^{(k)}\right)_{k \in \mathbb{N}}$. For an arbitrary subset $X \subseteq E$ and an infinite jet $f=\left(f^{k}\right)_{k \in \mathbb{N}}$ we introduce the Taylor polynomial $\left(T_{y}^{n} f\right)^{k}: X \rightarrow L_{\text {sym }}^{k}(E, F)$ of order $n$ at the point $y$ as follows:

$$
\left(T_{y}^{n} f\right)^{k}(x)\left(v_{1}, \ldots, v_{k}\right):=\sum_{j=0}^{n} \frac{1}{j !} f^{j+k}(y)\left(x-y, \ldots, x-y, v_{1}, \ldots, v_{k}\right) .
$$

The remainder is given by

$$
\left(R_{y}^{n} f\right)^{k}(x):=f^{k}(x)-\left(T_{y}^{n} f\right)^{k}(x)=\left(T_{x}^{n} f\right)^{k}(x)-\left(T_{y}^{n} f\right)^{k}(x)
$$

and so $\left(R_{y}^{n} f\right)^{k}(x) \in L_{\text {sym }}^{k}(E, F)$. We put now

$$
\|f\|_{k}:=\sup \left\{\left\|f^{k}(x)\right\|_{L_{\text {sym }}^{k}(E, F)}: x \in K\right\}
$$

and

$$
\left|\|f \mid\|_{n, k}:=\sup \left\{(n+1) ! \frac{\left\|\left(R_{y}^{n} f\right)^{k}(x)\right\|_{L_{s y m}^{k}(E, F)}}{\|x-y\|^{n+1}}: x, y \in K, x \neq y\right\} .\right.
$$

We supply $\mathcal{E}(U, F)$ with the seminorms $f \mapsto\left\|\left.j^{\infty}(f)\right|_{K}\right\|_{k}$, where $K \subseteq U$ is a compact set and $k \in \mathbb{N}$. If $K \subseteq E$ is compact and convex, then we introduce the space $\mathcal{E}(E \supseteq K, F)$ of Whitney-jets on $K$ by

$\mathcal{E}(E \supseteq K, F):=\left\{f=\left(f^{k}\right)_{k \in \mathbb{N}} \in \prod_{k \in \mathbb{N}} \mathcal{C}\left(K, L_{\text {sym }}^{k}(E, F)\right):\left|\|f \mid\|_{n, k}<+\infty \forall n, k \in \mathbb{N}\right\}\right.$

and we supply these spaces with both seminorms $\|f\|_{k}$ and $\left|\|f \mid\|_{n, k}\right.$ for $k, n \in \mathbb{N}$. Finally recall [6, Lemma 3.1.]:

Lemma 3.2. Let $E$ and $F$ be Banach spaces and $K \subseteq E$ be a compact convex subset. Then $\mathcal{E}(E \supseteq K, F)$ is a Fréchet space.

\subsection{Classes of ultra-differentiable mappings defined by a weight matrix}

Let $\mathcal{M}:=\left\{M^{x}: x \in \Lambda\right\}$ be $(\mathcal{M}), E$ and $F$ be Banach spaces and $K \subseteq E$ a compact subset. Then, as in [6, 4.1.], for $x \in \Lambda$ and $h>0$ we define

$$
\mathcal{E}_{M^{x}, h}(E \supseteq K, F):=\left\{\left(f^{j}\right)_{j} \in \prod_{j \in \mathbb{N}} \mathcal{C}\left(K, L_{\mathrm{sym}}^{j}(E, F)\right):\|f\|_{M^{x}, h}^{J}<+\infty\right\},
$$


where

$$
\|f\|_{M^{x}, h}^{J}:=\max \left\{\sup \left\{\frac{\|f\|_{k}}{h^{k} M_{k}^{x}}: k \in \mathbb{N}\right\}, \sup \left\{\frac{\left|\|f \mid\|_{n, k}\right.}{h^{n+k-1} M_{n+k+1}^{x}}: k, n \in \mathbb{N}\right\}\right\} .
$$

For open $U \subseteq E$ and compact $K \subseteq U$ we introduce the space

$$
\mathcal{E}_{M^{x}, K, h}(U, F):=\left\{f \in \mathcal{E}(U, F):\left.j^{\infty}(f)\right|_{K} \in \mathcal{E}_{M^{x}, h}(E \supseteq K, F)\right\},
$$

with semi-norm $f \mapsto\left\|\left.j^{\infty}(f)\right|_{K}\right\|_{M^{x}, h}^{J}$. It is not Hausdorff and for infinite dimensional $E$ its Hausdorff quotient will not always be complete. Note that if $K$ is assumed to be convex, then we can take on $\mathcal{E}_{M^{x}, K, h}(U, F)$ also the semi-norm

$$
f \mapsto \sup \left\{\frac{\left\|f^{(n)}(a)\right\|_{L_{\text {sym }}^{n}(E, F)}}{h^{n} M_{n}^{x}}: a \in K, n \in \mathbb{N}\right\}=:\|f\|_{M^{x}, K, h}^{J} .
$$

Thus we see that $\mathcal{E}_{M^{x}, K, h}(U, F)=\left\{f \in \mathcal{E}(U, F):\left(\left\|\left.j^{\infty}(f)\right|_{K}\right\|_{k}\right)_{k} \in \mathcal{F}_{M^{x}, h}\right\}$ holds with

$$
\mathcal{F}_{M^{x}, h}:=\left\{\left(f_{k}\right)_{k} \in \mathbb{R}_{>0}^{\mathbb{N}}: \exists C>0: \forall k \in \mathbb{N}:\left|f_{k}\right| \leq C h^{k} M_{k}^{x}\right\} .
$$

The bounded sets $\mathcal{B}$ in $\mathcal{E}_{M^{x}, K, h}(U, F)$ are exactly those $\mathcal{B} \subseteq \mathcal{E}(U, F)$ such that $\left(b_{m}\right)_{m} \in \mathcal{F}_{M^{x}, h}$ with $b_{m}:=\sup \left\{\left\|\left.j^{\infty}(f)\right|_{K}\right\|_{m}: f \in \mathcal{B}\right\}$.

Let $U \subseteq E$ be convex open and $K \subseteq U$ be convex compact, then define

$$
\begin{aligned}
& \mathcal{E}_{(\mathcal{M})}(E \supseteq K, F):=\lim _{x \in \overleftarrow{\Lambda, h}>0} \mathcal{E}_{M^{x}, h}(E \supseteq K, F) \\
& \mathcal{E}_{\{\mathcal{M}\}}(E \supseteq K, F):=\lim _{x \in \overrightarrow{\Lambda, h}>0} \mathcal{E}_{M^{x}, h}(E \supseteq K, F)
\end{aligned}
$$

and finally

$$
\mathcal{E}_{[\mathcal{M}]}(U, F):=\lim _{K \subseteq U} \mathcal{E}_{[\mathcal{M}]}(E \supseteq K, F),
$$

i.e.

$$
\mathcal{E}_{[\mathcal{M}]}(U, F):=\left\{f \in \mathcal{E}(U, F): \forall K:\left(\left.f^{(k)}\right|_{K}\right) \in \mathcal{E}_{[\mathcal{M}]}(E \supseteq K, F)\right\},
$$

where $K$ runs through all compact and convex subsets of $U$.

If $\Lambda=\mathbb{R}_{>0}$, then we can restrict in both cases to the countable diagonal, see also [9, 4.2.-4.4.]. We have $\mathcal{E}_{(\mathcal{M})}(E \supseteq K, F)=\lim _{n \in \mathbb{N}_{>0}} \mathcal{E}_{M^{1 / n}, 1 / n}(E \supseteq K, F)$ and $\mathcal{E}_{\{\mathcal{M}\}}(E \supseteq K, F)=\lim _{n \in \mathbb{N}_{>0}} \mathcal{E}_{M^{n}, n}(E \supseteq K, F)$.

As already mentioned in [6, Proposition 4.1. (3)] the space $\mathcal{E}_{\{\mathcal{M}\}}(E \supseteq K, F)$ is not a Silva space for infinite dimensional $E$, because the connecting mappings in the inductive limit $\lim _{x \in \widehat{\Lambda, h}>0} \mathcal{E}_{M^{x}, h}(E \supseteq K, F)$ are not compact any more. The set $\mathcal{B}:=\left\{\alpha \in E^{\prime}:\|\alpha\| \leq 1\right\}$ is bounded in $\mathcal{E}_{M^{k}, k}(E \supseteq K, \mathbb{R})$ for each $k \geq 1$. We have 
$\|\alpha\|_{0}=\sup \{|\alpha(x)|: x \in K\} \leq \sup \{\|x\|: x \in K\},\|\alpha\|_{1}=\|\alpha\| \leq 1$ and $\|\alpha\|_{m}=0$ for each $m \geq 2$. Moreover $\left(R_{y}^{n} \alpha\right)^{k}=0$ for $n+k \geq 1$ and $\left(R_{y}^{0} \alpha\right)^{0}=\alpha(x-y)$. But $\mathcal{B}$ is not relatively compact in any $\mathcal{E}_{M^{k}, k}(E \supseteq K, \mathbb{R}), k \geq 1$, because it is not even pointwise relatively compact in $\mathcal{C}(K, L(E, \mathbb{R}))$.

Moreover we define

$$
\begin{aligned}
& \mathcal{E}_{(\mathcal{M}), K}(U, F):=\lim _{x \in \overleftarrow{\Lambda, h}>0} \mathcal{E}_{M^{x}, K, h}(U, F) \\
& \mathcal{E}_{\{\mathcal{M}\}, K}(U, F):=\lim _{x \in \Lambda, h>0} \mathcal{E}_{M^{x}, K, h}(U, F)
\end{aligned}
$$

and so

$$
\begin{aligned}
& \mathcal{E}_{(\mathcal{M}), K}(U, F)=\left\{f \in \mathcal{E}(U, F):\left(\left\|\left.j^{\infty}(f)\right|_{K}\right\|_{k}\right)_{k} \in \mathcal{F}_{(\mathcal{M})}\right\} \\
& \mathcal{E}_{\{\mathcal{M}\}, K}(U, F)=\left\{f \in \mathcal{E}(U, F):\left(\left\|\left.j^{\infty}(f)\right|_{K}\right\|_{k}\right)_{k} \in \mathcal{F}_{\{\mathcal{M}\}}\right\}
\end{aligned}
$$

with $\mathcal{F}_{(\mathcal{M})}=\bigcap_{x \in \Lambda, h>0} \mathcal{F}_{M^{x}, h}, \mathcal{F}_{\{\mathcal{M}\}}=\bigcup_{x \in \Lambda, h>0} \mathcal{F}_{M^{x}, h}$.

The bounded sets $\mathcal{B} \subseteq \mathcal{E}_{[\mathcal{M}], K}(U, F)$ are exactly those $\mathcal{B} \subseteq \mathcal{E}(U, F)$ for which the sequence $\left(b_{m}\right)_{m}, b_{m}:=\sup \left\{\left\|\left.j^{\infty}(f)\right|_{K}\right\|_{m}: f \in \mathcal{B}\right\}$, belongs to $\mathcal{F}_{[\mathcal{M}]}$.

Finally we introduce

$$
\varliminf_{K \subseteq U} \mathcal{E}_{[\mathcal{M}], K}(U, F)=\left\{f \in \mathcal{E}(U, F): \forall K:\left(\left\|\left.j^{\infty}(f)\right|_{K}\right\|_{m}\right)_{m} \in \mathcal{F}_{[\mathcal{M}]}\right\} .
$$

The next result generalizes [6, Proposition 4.1.].

Proposition 3.4. Let $\mathcal{M}$ be $(\mathcal{M})$ with $\Lambda=\mathbb{R}_{>0}$, then the following completeness properties are valid:

(1) $\mathcal{E}_{M^{x}, h}(E \supseteq K, F)$ is a Banach space.

(2) $\mathcal{E}_{(\mathcal{M})}(E \supseteq K, F)$ is a Fréchet space.

(3) $\mathcal{E}_{\{\mathcal{M}\}}(E \supseteq K, F)$ is a compactly regular $(L B)$-space, i.e. compact subsets are contained and compact in some step and so $\left(c^{\infty}\right)$-complete, webbed and ultrabornological.

$(4) \mathcal{E}_{(\mathcal{M})}(U, F)$ and $\mathcal{E}_{\{\mathcal{M}\}}(U, F)$ are complete.

(5) As locally convex vector spaces we have

$$
\mathcal{E}_{(\mathcal{M})}(U, F)=\lim _{\overleftarrow{K \subseteq U}} \mathcal{E}_{(\mathcal{M})}(E \supseteq K, F)=\lim _{\overleftarrow{K \subseteq U}} \mathcal{E}_{(\mathcal{M}), K}(U, F)
$$

and

$$
\mathcal{E}_{\{\mathcal{M}\}}(U, F)=\lim _{\overleftarrow{K \subseteq U}} \mathcal{E}_{\{\mathcal{M}\}}(E \supseteq K, F)=\lim _{\overleftarrow{K \subseteq U}} \mathcal{E}_{\{\mathcal{M}\}, K}(U, F) .
$$


Proof. (1) This was already shown in [6, Proposition 4.1. (1)].

(2) Holds since $\Lambda=\mathbb{R}_{>0}$.

(3) We can restrict to $\Lambda=\mathbb{N}_{>0}$ and proceed analogously as in [6, Proposition 4.1. (3)]. To show that the inductive limit is compactly regular it suffices to show that there exists a sequence of increasing 0-neighborhoods $U_{n} \in \mathcal{E}_{M^{n}, n}(E \supseteq K, F)$ such that for each $n \in \mathbb{N}$ there exists $l \in \mathbb{N}$ with $l \geq n$ and for which the topologies of $\mathcal{E}_{M^{l}, l}(E \supseteq K, F)$ and of $\mathcal{E}_{M^{k}, k}(E \supseteq K, F)$ coincide on $U_{n}$ for all $k \geq l$.

In general, for indices $x_{1} \geq x_{2}$ and positive real numbers $h_{1} \geq h_{2}$ we have clearly by definition $\|\cdot\|_{M^{x_{1}, h_{1}}}^{J} \leq\|\cdot\|_{M^{x_{2}, h_{2}}}^{J}$. Consider now the $\varepsilon$-Ball $U_{\varepsilon}^{x, h}(f):=$ $\left\{g:\|g-f\|_{M^{x}, h}^{J} \leq \varepsilon\right\}$ in $\mathcal{E}_{M^{x}, h}(E \supseteq K, F)$ and we restrict to the diagonal $x=h=n$ and identify $U^{n, n}$ with $U^{n}$.

We show that for arbitrary $n \in \mathbb{N}_{>0}$ and $n_{2}>n_{1}:=2 n$, for each $\varepsilon>0$ and $f \in U_{1}^{n}(0)$ there exists $\delta>0$ such that $U_{\delta}^{n_{2}}(f) \cap U_{1}^{n}(0) \subseteq U_{\mathcal{\varepsilon}}^{n_{1}}(f)$.

By assumption $f \in U_{1}^{n}(0)=U_{1}^{n, n}(0)$ we have $\|f\|_{a} \leq n^{a} M_{a}^{n}$ and $\|f\| \|_{a, b} \leq$ $n^{a+b+1} M_{a+b+1}^{n}$ for all $a, b \in \mathbb{N}$. Consider $g \in U_{\delta}^{n_{2}}(f) \cap U_{1}^{n}(0)=U_{\delta}^{n_{2}, n_{2}}(f) \cap$ $U_{1}^{n, n}(0)$, then $\|g\|_{a} \leq n^{a} M_{a}^{n},\|g\|_{a, b} \leq n^{a+b+1} M_{a+b+1}^{n}$ and moreover $\|g-f\|_{a} \leq$ $\delta n_{2}^{a} M_{a}^{n_{2}},\left.\|g-f\|\right|_{a, b} \leq \delta n_{2}^{a+b+1} M_{a+b+1}^{n_{2}}$ for all $a, b \in \mathbb{N}$. We estimate similarly as in [6, Proposition 4.1. (3)]. So for given $\varepsilon>0$ consider $N \in \mathbb{N}$ (minimal) with $\frac{1}{2^{N}}<\frac{\varepsilon}{2}$ and put $\delta:=\varepsilon\left(\frac{n_{1}}{n_{2}}\right)^{N-1} \frac{1}{M_{N}^{n_{2}}}$.

For $a \geq N$ we have $\frac{1}{2^{a}} \leq \frac{1}{2^{N}}<\frac{\varepsilon}{2}(\star)$, so use triangle-inequality to get

$$
\|g-f\|_{a} \leq\|g\|_{a}+\|f\|_{a} \leq 2 n^{a} M_{a}^{n}=2 n_{1}^{a} M_{a}^{n} \frac{1}{2^{a}} \underbrace{<}_{(\star)} \varepsilon n_{1}^{a} M_{a}^{n} \leq \varepsilon n_{1}^{a} M_{a}^{n_{1}}
$$

and the last inequality holds since $n_{1}=2 n>n$ and so $M_{a}^{n} \leq M_{a}^{n_{1}}$ for all $a \in \mathbb{N}$. For $a<N$ we have

$$
\|g-f\|_{a} \leq \delta n_{2}^{a} M_{a}^{n_{2}} \leq \varepsilon n_{1}^{a} \frac{M_{a}^{n_{2}}}{M_{N}^{n_{2}}} \leq \varepsilon n_{1}^{a} \leq \varepsilon n_{1}^{a} M_{a}^{n_{1}}
$$

because $M_{a}^{n} \leq M_{N^{\prime}}^{n}\left(\frac{n_{1}}{n_{2}}\right)^{N-1} \leq\left(\frac{n_{1}}{n_{2}}\right)^{a}$ since $a<N, \frac{n_{1}}{n_{2}}<1$ and finally $M_{a}^{n_{1}} \geq 1$. Analogously we can use the same estimates for $\left.\|\cdot\|\right|_{a, b}$ instead of $\|\cdot\|_{a}$ for each $a, b \in \mathbb{N}$.

(4) In the Beurling-case we have a projective limit of Fréchet spaces, in the Roumieucase a projective limit of ( $L B)$-spaces, which are all compactly regular by (3) and so complete, too. Since projective limits of complete spaces are complete we are done.

(5) This holds precisely by the same proof as given in [6, Proposition 4.1. (5)] 
Let $E, F$ be convenient, $U \subseteq E$ be $c^{\infty}$-open, then define

$$
\begin{aligned}
\mathcal{E}_{(\mathcal{M})}^{\mathrm{b}}(U, F):= & \left\{f \in \mathcal{E}(U, F): \forall B: \forall K \subseteq U \cap E_{B}: \forall x \in \Lambda \forall h>0:\right. \\
& \left.\left\{\frac{f^{(k)}(a)\left(v_{1}, \ldots, v_{k}\right)}{h^{k} M_{k}^{x}}: k \in \mathbb{N}, a \in K,\left\|v_{i}\right\|_{B} \leq 1\right\} \text { is bounded in } F\right\} \\
& =\left\{f \in \mathcal{E}(U, F): \forall B: \forall K \subseteq U \cap E_{B}: \forall x \in \Lambda \forall h>0:\right. \\
& \left.\left\{\frac{d_{v}^{k} f(a)\left(v_{1}, \ldots, v_{k}\right)}{h^{k} M_{k}^{x}}: k \in \mathbb{N}, a \in K,\left\|v_{i}\right\|_{B} \leq 1\right\} \text { is bounded in } F\right\} .
\end{aligned}
$$

and

$$
\begin{aligned}
\mathcal{E}_{\{\mathcal{M}\}}^{\mathrm{b}}(U, F):= & \left\{f \in \mathcal{E}(U, F): \forall B: \forall K \subseteq U \cap E_{B}: \exists x \in \Lambda \exists h>0:\right. \\
& \left.\left\{\frac{f^{(k)}(a)\left(v_{1}, \ldots, v_{k}\right)}{h^{k} M_{k}^{x}}: k \in \mathbb{N}, a \in K,\left\|v_{i}\right\|_{B} \leq 1\right\} \text { is bounded in } F\right\} \\
& =\left\{f \in \mathcal{E}(U, F): \forall B: \forall K \subseteq U \cap E_{B}: \exists x \in \Lambda \exists h>0:\right. \\
& \left.\left\{\frac{d_{v}^{k} f(a)\left(v_{1}, \ldots, v_{k}\right)}{h^{k} M_{k}^{x}}: k \in \mathbb{N}, a \in K,\left\|v_{i}\right\|_{B} \leq 1\right\} \text { is bounded in } F\right\} .
\end{aligned}
$$

$B$ runs through all closed absolutely convex bounded subsets in $E, E_{B}$ is the complete vector space generated by $B$ with the Minkowski-functional $\|\cdot\|_{B}$. Finally $K$ runs through all sets in $U \cap E_{B}$ which are compact w.r.t. the norm $\|\cdot\|_{B}$. If $E$ and $F$ both are Banach spaces and $U \subseteq E$ open we have $\mathcal{E}_{[\mathcal{M}]}^{\mathrm{b}}(U, F)=\mathcal{E}_{[\mathcal{M}]}(U, F)$, where the latter space is introduced in (3.1).

Now we give the most important definition:

$$
\mathcal{E}_{[\mathcal{M}]}(U, F):=\left\{f \in \mathcal{E}(U, F): \forall \alpha \in F^{*}: \forall B: \alpha \circ f \circ i_{B} \in \mathcal{E}_{[\mathcal{M}]}\left(U_{B}, \mathbb{R}\right)\right\},
$$

where $B$ is running again through all closed absolutely convex bounded subsets in $E$, the mapping $i_{B}: E_{B} \rightarrow E$ denotes the inclusion of $E_{B}$ in $E$ and we write $U_{B}:=i_{B}^{-1}(U)$. The initial locally convex structure is now induced by all linear mappings

$$
\mathcal{E}_{[\mathcal{M}]}\left(i_{B}, \alpha\right): \mathcal{E}_{[\mathcal{M}]}(U, F) \longrightarrow \mathcal{E}_{[\mathcal{M}]}\left(U_{B}, \mathbb{R}\right), \quad f \mapsto \alpha \circ f \circ i_{B} .
$$

$\mathcal{E}_{[\mathcal{M}]}(U, F) \subseteq \prod_{\alpha, B} \mathcal{E}_{[\mathcal{M}]}\left(U_{B}, \mathbb{R}\right)$ are convenient vector spaces as $c^{\infty}$-closed subspaces in the product: Smoothness can be tested by composing with inclusions $E_{B} \rightarrow E$ and $\alpha \in F^{*}$ as mentioned in [4, 2.14.4, 1.8]. Hence we obtain the representation

$$
\mathcal{E}_{[\mathcal{M}]}(U, F):=\left\{f \in F^{U}: \forall \alpha \in F^{*} \forall B: \alpha \circ f \circ i_{B} \in \mathcal{E}_{[\mathcal{M}]}\left(U_{B}, \mathbb{R}\right)\right\} .
$$

All definitions given here are clearly generalizations of the definitions in [6, 4.2.] for constant matrices. 


\section{Projective descriptions for $\mathcal{E}_{[\mathcal{M}]}$}

In this section we are going to study one of the most important new techniques in this work. Using abstract families of sequences of positive real numbers we prove projective representations for the Roumieu-class $\mathcal{E}_{\{\mathcal{M}\}}$. This technique is very important since we want to get rid of both existence quantifiers in the definitions of $\mathcal{E}_{\{\mathcal{M}\}}$ so we want to generalize [6, Lemma 4.6.]. Furthermore we are going to prove analogous results for the Beurling-case $\mathcal{E}_{(\mathcal{M})}$ and generalize [6, Lemma 4.5.]. To do so we have to show variations and generalizations of [4, Lemma 9.2.] (for the Roumieu-case) and of the Lemma between Lemma 4.5. and Lemma 4.6. in [6] (for the Beurling-case).

We will obtain different projective representations for $\mathcal{E}_{[\mathcal{M}]}$. The choice of the appropriate representation depends on the application in the proofs. To show closedness under composition in section 5, see Theorem 5.8 and Theorem 5.9, we will have to use the versions using the Fa-di-Bruno-property $\left(\mathcal{M}_{[\mathrm{FdB}]}\right)$. For the exponential laws in section 6 the versions only assuming $(\mathcal{M})$ or $\left(\mathcal{M}_{\text {sc }}\right)$ for $\mathcal{M}$ are sufficient.

First we have to introduce several classes of sequences of positive real numbers $\left(r_{k}\right)_{k}$ and $\left(s_{k}\right)_{k}$. It is no restriction to assume $r_{0}=1$ resp. $s_{0}=1$ (normalization) for all occurring sequences.

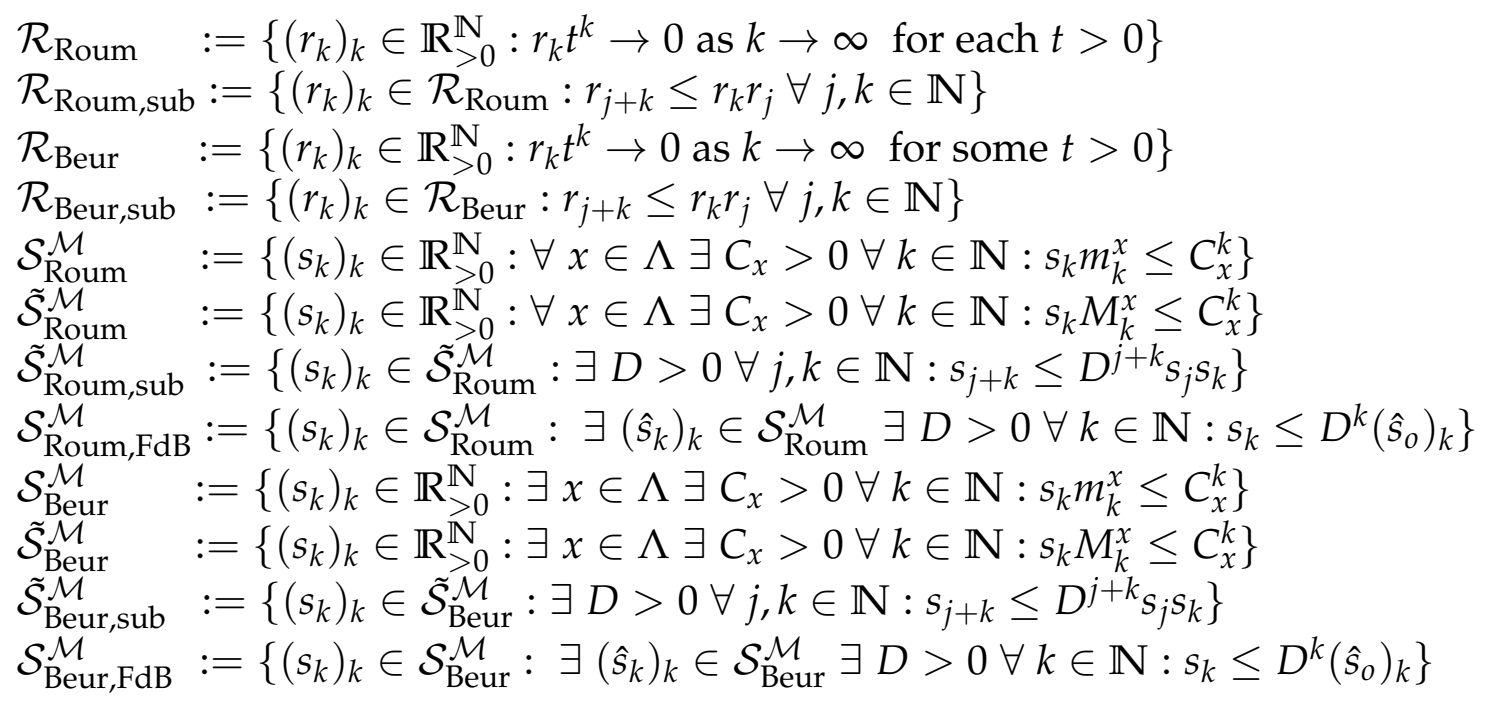

For $\left(s_{k}\right)_{k} \in \mathcal{S}_{\text {Roum }}^{\mathcal{M}} \mathcal{S}_{\text {Beur }}^{\mathcal{M}}$ we have put

$$
\left(s_{o}\right)_{k}:=\min \left\{s_{j} s_{\alpha_{1}} \cdots s_{\alpha_{j}}: \alpha_{i} \in \mathbb{N}_{>0}, \alpha_{1}+\cdots+\alpha_{j}=k\right\}, \quad\left(s_{o}\right)_{0}:=1 .
$$

By definition $\tilde{\mathcal{S}}_{\text {Roum }}^{\mathcal{M}} \subseteq \mathcal{S}_{\text {Roum }}^{\mathcal{M}}$ and $\left(s_{k}\right)_{k} \in \tilde{\mathcal{S}}_{\text {Roum }}^{\mathcal{M}}$ if and only if $\left(k ! s_{k}\right)_{k} \in \mathcal{S}_{\text {Roum }}^{\mathcal{M}}$ respectively for the Beurling-case. If $\left(s_{k}\right)_{k} \in \mathcal{S}_{\text {Beur }}^{\mathcal{M}}, \mathcal{S}_{\text {Beur }}^{\mathcal{M}}$ holds for $x \in \Lambda$, then also for all $y \leq x$, too. All occurring sets are stable w.r.t. $\left({ }_{k}\right)_{k} \mapsto\left(B^{k} \cdot{ }_{k}\right)_{k}$ for arbitrary $B>0$.

Using [6, Lemma 4.6.] directly we get:

Proposition 4.1. Let $\mathcal{M}=\left\{M^{x}: x \in \Lambda\right\}$ be $(\mathcal{M})$, E, $F$ be Banach spaces, $U \subseteq E$ open and $f: U \rightarrow F$ a $\mathcal{E}$-mapping. Then the following are equivalent:

(1) $f$ is $\mathcal{E}_{\{\mathcal{M}\}}=\mathcal{E}_{\{\mathcal{M}\}}^{\mathrm{b}}$. 
(2) For each compact $K \subseteq U$ there exists $x \in \Lambda$ such that for each $\left(r_{k}\right)_{k} \in \mathcal{R}_{\text {Roum }}$

$$
\left\{\frac{f^{(k)}(a)\left(v_{1}, \ldots, v_{k}\right) r_{k}}{M_{k}^{x}}: a \in K, k \in \mathbb{N},\left\|v_{i}\right\|_{E} \leq 1\right\}
$$

is bounded in F.

(3) For each compact $K \subseteq U$ there exists $x \in \Lambda$ such that for each $\left(r_{k}\right)_{k} \in \mathcal{R}_{\text {Roum,sub }}$ there exists $\varepsilon>0$ such that

$$
\left\{\frac{f^{(k)}(a)\left(v_{1}, \ldots, v_{k}\right) r_{k} \varepsilon^{k}}{M_{k}^{x}}: a \in K, k \in \mathbb{N},\left\|v_{i}\right\|_{E} \leq 1\right\}
$$

is bounded in F.

Note that $\mathcal{E}_{\{\mathcal{M}\}}=\mathcal{E}_{\{\mathcal{M}\}}^{\mathrm{b}}$ holds by Lemma 5.4 below, but for our approach in this work we also have to get rid of the second existence quantifier.

\subsection{Roumieu-case with $\left(\mathcal{M}_{\{\mathrm{FdB}\}}\right)$}

We prove the following generalization of [4, Lemma 9.2.]:

Lemma 4.3. Let $\mathcal{M}=\left\{M^{x}: x \in \Lambda\right\}$ be $\left(\mathcal{M}_{\mathrm{sc}}\right)$ with $\Lambda=\mathbb{N}_{>0}$ and $\left(\mathcal{M}_{\{\mathrm{FdB}\}}\right)$. For a formal power series $\sum_{k \geq 0} a_{k}^{x} t^{k}=\sum_{k \geq 0} \frac{b_{k}}{k ! m_{k}^{x}} t^{k}$, so $a_{k}^{x}:=\frac{b_{k}}{M_{k}^{x}}$, the following are equivalent:

(1) There exists $x \in \Lambda$ such that $\sum_{k \geq 0} a_{k}^{x} t^{k}$ has positive radius of convergence.

(2) $\sum_{k \geq 0} \frac{b_{k} r_{k} s_{k}}{k !}$ converges absolutely for all $\left(r_{k}\right)_{k} \in \mathcal{R}_{\text {Roum }}$ and $\left(s_{k}\right)_{k} \in \mathcal{S}_{\text {Roum }}^{\mathcal{M}}$

(3) The sequence $\left(\frac{b_{k} r_{k} s_{k}}{k !}\right)_{k}$ is bounded for all $\left(r_{k}\right)_{k} \in \mathcal{R}_{\text {Roum }}$ and $\left(s_{k}\right)_{k} \in \mathcal{S}_{\text {Roum }}^{\mathcal{M}}$

(4) For each $\left(r_{k}\right)_{k} \in \mathcal{R}_{\text {Roum,sub }}$ and for each $\left(s_{k}\right)_{k} \in \mathcal{S}_{\text {Roum,FdB }}^{\mathcal{M}}$ there exists $\varepsilon>0$ such that $\left(\frac{b_{k}{ }^{r} s_{k}}{k !} \varepsilon^{k}\right)_{k}$ is bounded.

Proof. (1) $\Rightarrow(2)$ For the given series ( $x \in \Lambda$ coming from (1)) and arbitrary $\left(r_{k}\right)_{k}$ and $\left(s_{k}\right)_{k}$ as considered in (2) we have

$$
\sum_{k \geq 0} \frac{b_{k} r_{k} s_{k}}{k !}=\sum_{k \geq 0} a_{k}^{x} m_{k}^{x} r_{k} s_{k}=\sum_{k \geq 0}\left(a_{k}^{x} t^{k}\right) \underbrace{\left(s_{k} m_{k}^{x}\right)}_{\leq C_{x}^{k}} \frac{r_{k}}{t^{k}} \leq \sum_{k \geq 0}\left(a_{k}^{x} t^{k}\right) \underbrace{r_{k}\left(\frac{C_{x}}{t}\right)^{k}}_{\rightarrow 0, \text { as } k \rightarrow \infty},
$$

hence the first sum converges for $t>0$ sufficiently small.

(2) $\Rightarrow(3) \Rightarrow(4)$ are clearly satisfied.

$(4) \Rightarrow(1)$ Since $\left(\mathcal{M}_{\{\mathrm{FdB}\}}\right)$ is satisfied and $m^{x} \leq m^{y}$ for $x \leq y$ we can associate to each $x \in \Lambda$ the index $\alpha(x):=\min \left\{y \in \Lambda:\left(m^{x}\right)^{\circ} \llbracket m^{y}\right\}$. Since $\left(m^{x}\right)^{\circ} \leq\left(m^{y}\right)^{\circ}$ for $x \leq y$ we also have $\alpha(x) \leq \alpha(y)$ for such indices and $\lim _{x \rightarrow \infty} \alpha(x)=+\infty$. 
On the other hand for $y \geq \alpha(1)$ we can define $\beta(y):=\max \{x \in \Lambda: \alpha(x) \leq y\}$ which is clearly well-defined. So $\beta\left(y_{1}\right) \leq \beta\left(y_{2}\right)$ for $y_{1} \leq y_{2}, \lim _{y \rightarrow \infty} \beta(y)=+\infty$ and finally by construction for each $x \in \mathbb{N}_{>0}, x \geq \alpha(1)$, there exist $y \in \mathbb{N}_{>0}$, $y \leq x$, with $\left(m^{y}\right)^{q} \preceq m^{x}$. Note that this does not imply $\left(\mathcal{M}_{(\mathrm{FdB})}\right)$. W.l.o.g. we could assume that $\alpha(x)=x+1$ and so $\beta(y)=y-1$. If $\mathcal{M}$ has in addition $\left(\mathcal{M}_{\left\{\mathcal{C}^{\omega}\right\}}\right)$, i.e. the real analytic functions are contained in $\mathcal{E}_{\{\mathcal{M}\}}$, then we can take w.l.o.g. $M^{1}=(p !)_{p \in \mathbb{N}}$, so $m_{p}^{1}=1$ for each $p$ and $\alpha(1)=1$.

We prove by contradiction. So assume that each $\sum_{k \geq 0} a_{k}^{x} t^{k}$ would have radius of convergence 0 . Then we would get $\sum_{k \geq 0}\left|a_{k}^{x}\right|\left(\frac{1}{n^{2}}\right)^{k}=+\infty$ for each $n \in \mathbb{N}_{>0}$ and each $x \in \Lambda=\mathbb{N}_{>0}$. Consider now $n \in \mathbb{N}_{>0}$ and $x:=n+\alpha(1)$ and so we find an increasing sequence $\left(k_{n}\right)_{n \geq 0}$ with $k_{0}=1, \lim _{n \rightarrow \infty} k_{n}=+\infty$ such that

$$
\forall n \in \mathbb{N}_{>0}: \sum_{k=k_{n-1}}^{k_{n}-1}\left|a_{k}^{n+\alpha(1)}\right|\left(\frac{1}{n^{2}}\right)^{k} \geq 1
$$

We put now

$$
r_{k}:=\left(\frac{1}{n^{2}}\right)^{k} \text { for } k_{n-1} \leq k \leq k_{n}-1, n \in \mathbb{N}_{>0}
$$

and show $\left(r_{k}\right)_{k} \in \mathcal{R}_{\text {Roum,sub }}$. For $k_{n-1} \leq k \leq k_{n}-1$ by definition $r_{k} t^{k}=\left(\frac{t}{n^{2}}\right)^{k}$, and so $r_{k} t^{k} \rightarrow 0$ as $k \rightarrow \infty$ and all $t>0$. Clearly $\left(r_{k}\right)_{k}$ is also log-sub-additive. In addition one can see that $\left(\sqrt{r_{k}}\right)_{k} \in \mathcal{R}_{\text {Roum,sub }}$ and so for all $\varepsilon>0$ there exists $k_{\varepsilon} \in \mathbb{N}$ such that for all $k \geq k_{\varepsilon}$ we have $\sqrt{r_{k}} \frac{1}{\varepsilon^{k}} \leq 1 \Leftrightarrow \sqrt{r_{k}} \leq \varepsilon^{k}$.

No we define $s:=\left(s_{k}\right)_{k}$. We put $s_{k}:=\frac{1}{m_{k}^{\gamma(k)}}$, where $\gamma(k):=n+\alpha(1)$ for $k_{n-1} \leq k \leq k_{n}-1, n \in \mathbb{N}_{>0}$, and show $\left(s_{k}\right)_{k} \in \mathcal{S}_{\text {Roum,FdB }}^{\mathcal{M}}$

So let $x \in \Lambda$ be arbitrary (large) but fixed, then for $k_{n-1} \leq k \leq k_{n}-1$ we get $s_{k} m_{k}^{x}=\frac{m_{k}^{x}}{m_{k}^{\gamma(k)}}=\frac{M_{k}^{x}}{M_{k}^{\gamma(k)}}$. For all $k \in \mathbb{N}$ we can estimate $\frac{M_{k}^{x}}{M_{k}^{\gamma(k)}} \leq C_{x}^{k}$ with some constant $C_{x}>0$, because $\lim _{k \rightarrow \infty} \gamma(k)=+\infty$. This proves $\left(s_{k}\right)_{k} \in \mathcal{S}_{\text {Roum }}^{\mathcal{M}}$. Define

$$
\hat{s}_{k}:=\frac{1}{m_{k}^{\beta(\gamma(k))}} \quad \text { for } k_{n-1} \leq k \leq k_{n}-1, n \in \mathbb{N}_{>0} \text {, }
$$

and similarly we find a constant $D_{x}>0$ such that $\hat{s}_{k} m_{k}^{x} \leq D_{x}^{k}$ for each $x \in \Lambda$ and $k \in \mathbb{N}$ because $\lim _{k \rightarrow \infty} \beta(\gamma(k))=+\infty$. This proves $\left(\hat{s}_{k}\right)_{k} \in \mathcal{S}_{\text {Roum }}^{\mathcal{M}}$. For $\delta_{1}+\cdots+\delta_{j}=k$ we obtain for $k \in \mathbb{N}$ with $k_{n-1} \leq k \leq k_{n}-1, n \in \mathbb{N}_{>0}$ :

$$
\begin{aligned}
s_{k} & =\frac{1}{m_{k}^{\gamma(k)}} \leq C h^{k} \frac{1}{m_{j}^{\beta(\gamma(k))} m_{\delta_{1}}^{\beta(\gamma(k))} \cdots m_{\delta_{j}}^{\beta(\gamma(k))}} \\
& \leq C h^{k} \frac{1}{m_{j}^{\beta(\gamma(j))} m_{\delta_{1}}^{\beta\left(\gamma\left(\delta_{1}\right)\right)} \cdots m_{\delta_{j}}^{\beta\left(\gamma\left(\delta_{j}\right)\right)}}=C h^{k} \hat{s}_{j} \hat{s}_{\delta_{1}} \cdots \hat{s}_{\delta_{j}},
\end{aligned}
$$


which precisely shows $\left\{\underline{\underline{\Omega}} \hat{\beta}_{0}\right.$. The first inequality holds by $\left(\mathcal{M}_{\{\mathrm{FdB}\}}\right)$ and by definition of $\beta$, the second because $j, \delta_{1}, \ldots, \delta_{j} \leq k$. So $s$ is as desired.

Moreover

$$
\sum_{k \geq 1} \frac{\left|b_{k}\right| r_{k} s_{k}}{k !}=\sum_{n \geq 1} \sum_{k=k_{n-1}}^{k_{n}-1} \frac{\left|b_{k}\right| r_{k} s_{k}}{k !}=\sum_{n \geq 1} \sum_{k=k_{n-1}}^{k_{n}-1}\left|a_{k}^{n+\alpha(1)}\right|\left(\frac{1}{n^{2}}\right)^{k} \geq \sum_{n \geq 1} 1=+\infty,
$$

because by definition $\frac{\left|b_{k}\right|}{k !} s_{k}=\frac{\left|b_{k}\right|}{M_{k}^{n+\alpha(1)}}=\left|a_{k}^{n+\alpha(1)}\right|$ for $k_{n-1} \leq k \leq k_{n}-1$ (note that $n(k)=n+\alpha(1)$ for $\left.k \in\left[k_{n-1}, k_{n}-1\right]\right)$.

Finally we show that $\left(\frac{b_{k}}{k !} \sqrt{r_{k}} s_{k}(2 \varepsilon)^{k}\right)_{k}$ cannot be bounded for any $\varepsilon>0$. First we get

$$
\sum_{k \geq 1} \frac{\left|b_{k}\right|}{k !} \sqrt{r_{k}} s_{k} \varepsilon^{k} \geq \sum_{k \geq k_{\varepsilon}} \frac{\left|b_{k}\right|}{k !} \sqrt{r_{k}} s_{k} \underbrace{\varepsilon^{k}}_{\geq \sqrt{r_{k}}} \geq \sum_{k \geq k_{\varepsilon}} \frac{\left|b_{k}\right|}{k !} r_{k} s_{k}=+\infty .
$$

But if the sequence would be bounded for some $\varepsilon$, then for all $k \in \mathbb{N}$ we would get $\frac{b_{k}}{k !} \sqrt{r_{k}} s_{k} \varepsilon^{k} \leq \frac{C}{2^{k}}$, hence $\sum_{k \geq 0} \frac{\left|b_{k}\right|}{k !} \sqrt{r_{k}} s_{k} \varepsilon^{k} \leq \sum_{k \geq 0} \frac{C}{2^{k}}=2 C$, a contradiction.

We use Lemma 4.3 to generalize [6, Lemma 4.6.].

Proposition 4.4. Let $\mathcal{M}=\left\{M^{x}: x \in \Lambda\right\}$ be $\left(\mathcal{M}_{\mathrm{sc}}\right)$ with $\Lambda=\mathbb{N}_{>0}$ and $\left(\mathcal{M}_{\{\mathrm{FdB}\}}\right)$ Let $E, F$ be Banach spaces, $U \subseteq E$ open and $f: U \rightarrow F$ a $\mathcal{E}$-mapping. Then the following are equivalent:

(1) $f$ is $\mathcal{E}_{\{\mathcal{M}\}}=\mathcal{E}_{\{\mathcal{M}\}}^{\mathrm{b}}$.

(2) For each compact $K \subseteq U$, for each $\left(r_{k}\right)_{k} \in \mathcal{R}_{\text {Roum }}$ and each $\left(s_{k}\right)_{k} \in \mathcal{S}_{\text {Roum }}^{\mathcal{M}}$ the set

$$
\left\{\frac{f^{(k)}(a)\left(v_{1}, \ldots, v_{k}\right)}{k !} r_{k} s_{k}: a \in K, k \in \mathbb{N},\left\|v_{i}\right\|_{E} \leq 1\right\}
$$

is bounded in F.

(3) For each compact $K \subseteq U$, for each $\left(r_{k}\right)_{k} \in \mathcal{R}_{\text {Roum,sub }}$ and for each $\left(s_{k}\right)_{k} \in \mathcal{S}_{\mathrm{Roum}, \mathrm{FdB}}^{\mathcal{M}}$ there exists $\varepsilon>0$ such that the set

$$
\left\{\frac{f^{(k)}(a)\left(v_{1}, \ldots, v_{k}\right)}{k !} r_{k} s_{k} \varepsilon^{k}: a \in K, k \in \mathbb{N},\left\|v_{i}\right\|_{E} \leq 1\right\}
$$

is bounded in F.

Proof. (1) $\Rightarrow(2)$ Let $f$ be $\mathcal{E}_{\{\mathcal{M}\}}$ and $K \subseteq U$ compact, then estimate as follows (where we use Lemma5.4below):

$$
\left\|\frac{f^{(k)}(a)}{k !} r_{k} s_{k}\right\|_{L^{k}(E, F)}=\left\|\frac{f^{(k)}(a)}{k ! m_{k}^{x} h^{k}}\right\|_{L^{k}(E, F)}|r_{k} h^{k} \underbrace{s_{k} m_{k}^{x}}_{\leq C_{x}^{k}}| \leq\left\|\frac{f^{(k)}(a)}{h^{k} M_{k}^{x}}\right\|_{L^{k}(E, F)} \underbrace{\left|r_{k}\left(C_{x} h\right)^{k}\right|}_{\rightarrow 0, \text { as } k \rightarrow \infty}
$$


for $a \in K, x \in \Lambda$ and $h>0$ large enough (depending on $K$ and $f$ ) and for arbitrary $\left(r_{k}\right)_{k}$ and $\left(s_{k}\right)_{k}$ as considered in (2).

$(2) \Rightarrow$ (3) Take $\varepsilon=1$.

$(3) \Rightarrow(1)$ We use $(4) \Rightarrow(1)$ in Lemma 4.3 , Let $K \subseteq U$ be an arbitrary compact set but fixed and put $b_{k}:=\sup _{a \in K}\left\|f^{(k)}(a)\right\|_{L^{k}(E, F)}$. Then there exists $h>0$ and $x \in \Lambda$ such that $\sup _{k \in \mathbb{N}} \frac{b_{k}}{M_{x}^{k} h^{k}}<+\infty$, hence $f$ is $\mathcal{E}_{\{\mathcal{M}\}}$.

\subsection{Roumieu-case without $\left(\mathcal{M}_{\{\mathrm{FdB}\}}\right)$}

Lemma 4.6. Let $\mathcal{M}=\left\{M^{x}: x \in \Lambda\right\}$ be ( $\left.\mathcal{M}_{\mathrm{sc}}\right)$ with $\Lambda=\mathbb{N}_{>0}$. For a formal power series $\sum_{k \geq 0} a_{k}^{x} t^{k}=\sum_{k \geq 0} \frac{b_{k}}{M_{k}^{x}} t^{k}$ the following are equivalent:

(1) There exists $x \in \Lambda$ such that $\sum_{k \geq 0} a_{k}^{x} t^{k}$ has positive radius of convergence.

(2) $\sum_{k \geq 0} b_{k} r_{k} s_{k}$ converges absolutely for all $\left(r_{k}\right)_{k} \in \mathcal{R}_{\text {Roum }}$ and $\left(s_{k}\right)_{k} \in \tilde{\mathcal{S}}_{\text {Roum }}^{\mathcal{M}}$

(3) $\left(b_{k} r_{k} s_{k}\right)_{k}$ is bounded for all $\left(r_{k}\right)_{k} \in \mathcal{R}_{\text {Roum }}$ and $\left(s_{k}\right)_{k} \in \tilde{\mathcal{S}}_{\text {Roum,sub }}^{\mathcal{M}}$

(4) For each $\left(r_{k}\right)_{k} \in \mathcal{R}_{\text {Roum,sub }}$ and for each $\left(s_{k}\right)_{k} \in \tilde{\mathcal{S}}_{\text {Roum,sub }}^{\mathcal{M}}$ there exists $\varepsilon>0$ such that $\left(b_{k} r_{k} s_{k} \varepsilon^{k}\right)_{k}$ is bounded.

If $\mathcal{M}$ is $(\mathcal{M})$, then in (3) and (4) we replace $\tilde{\mathcal{S}}_{\text {Roum,sub }}^{\mathcal{M}}$ by $\tilde{\mathcal{S}}_{\text {Roum }}^{\mathcal{M}}$

Proof. $(1) \Rightarrow(2) \Rightarrow(3) \Rightarrow(4)$ is the same as in Lemma 4.3 . For $(4) \Rightarrow(1)$ we prove again by contradiction. In (4.1) consider $x=n \in \mathbb{N}_{>0}$, take the same $r=\left(r_{k}\right)_{k}$ and for $s=\left(s_{k}\right)_{k}$ we put $s_{k}:=\frac{1}{M_{k}^{n}}$ if $k_{n-1} \leq k \leq k_{n}-1$. If $\mathcal{M}$ is $\left(\mathcal{M}_{\mathrm{sc}}\right)$ then we have $M_{j}^{x} M_{k}^{x} \leq M_{j+k}^{x}$ for each $j, k \in \mathbb{N}$ and $x \in \Lambda$ and $M^{x} \leq M^{y}$ for $x \leq y$. This implies $\left(s_{k}\right)_{k} \in \tilde{\mathcal{S}}_{\text {Roum,sub }}^{\mathcal{M}}$ If $\mathcal{M}$ is $(\mathcal{M})$, then $\left(s_{k}\right)_{k} \in \tilde{\mathcal{S}}_{\text {Roum }}^{\mathcal{M}}$ holds by definition.

So we can prove a new version of Proposition 4.4 .

Proposition 4.7. Let $\mathcal{M}=\left\{M^{x}: x \in \Lambda\right\}$ be $\left(\mathcal{M}_{\mathrm{sc}}\right)$ with $\Lambda=\mathbb{N}_{>0}$. Let $E, F$ be Banach spaces, $U \subseteq$ E open and $f: U \rightarrow F$ a $\mathcal{E}$-mapping, then the following are equivalent:

(1) $f$ is $\mathcal{E}_{\{\mathcal{M}\}}=\mathcal{E}_{\{\mathcal{M}\}}^{\mathrm{b}}$.

(2) For each compact $K \subseteq U$, for each $\left(r_{k}\right)_{k} \in \mathcal{R}_{\text {Roum }}$ and for each $\left(s_{k}\right)_{k} \in \tilde{\mathcal{S}}_{\text {Roum }}^{\mathcal{M}}$ the set

$$
\left\{f^{(k)}(a)\left(v_{1}, \ldots, v_{k}\right) r_{k} s_{k}: a \in K, k \in \mathbb{N},\left\|v_{i}\right\|_{E} \leq 1\right\}
$$

is bounded in F.

(3) For each compact $K \subseteq U$, for each $\left(r_{k}\right)_{k} \in \mathcal{R}_{\text {Roum,sub }}$ and for each $\left(s_{k}\right)_{k} \in \tilde{\mathcal{S}}_{\text {Roum,sub }}^{\mathcal{M}}$ there exists $\varepsilon>0$ such that the set

$$
\left\{f^{(k)}(a)\left(v_{1}, \ldots, v_{k}\right) r_{k} s_{k} \varepsilon^{k}: a \in K, k \in \mathbb{N},\left\|v_{i}\right\|_{E} \leq 1\right\}
$$

is bounded in F. 
If $\mathcal{M}$ is $(\mathcal{M})$, then in (3) we replace $\tilde{\mathcal{S}}_{\text {Roum,sub }}^{\mathcal{M}}$ by $\tilde{\mathcal{S}}_{\text {Roum }}^{\mathcal{M}}$

Proof. Use precisely the same arguments as in Proposition 4.4, for (3) $\Rightarrow(1)$ we use $(4) \Rightarrow(1)$ in Lemma 4.6 .

\subsection{Beurling-case with $\left(\mathcal{M}_{(\mathrm{FdB})}\right)$}

Lemma 4.9. Let $\mathcal{M}=\left\{M^{x}: x \in \Lambda\right\}$ be $\left(\mathcal{M}_{\mathrm{sc}}\right)$ with $\Lambda=\mathbb{R}_{>0}$ and $\left(\mathcal{M}_{(\mathrm{FdB})}\right)$ For $a$ formal power series $\sum_{k \geq 0} a_{k}^{x} t^{k}=\sum_{k \geq 0} \frac{b_{k}}{M_{k}^{x}} t^{k}$, so $a_{k}^{x}:=\frac{b_{k}}{M_{k}^{x}}$, the following are equivalent:

(1) The series $\sum_{k \geq 0} a_{k}^{x} t^{k}$ has infinite radius of convergence for each $x \in \Lambda$.

(2) For each $\left(r_{k}\right)_{k} \in \overline{\mathcal{R}_{\text {Beur,sub }}}$ and for each $\left(s_{k}\right)_{k} \in \mathcal{S}_{\text {Beur,FdB }}^{\mathcal{M}}$ the sequence $\left(\frac{b_{k}}{k !} r_{k} s_{k} \delta^{k}\right)_{k}$ is bounded for each $\delta>0$.

Proof. (1) $\Rightarrow(2)$ Let $\left(r_{k}\right)_{k}$ and $\left(s_{k}\right)_{k}$ be given as considered in (2), then

$$
\sum_{k \geq 0} \frac{b_{k}}{k !} r_{k} s_{k} \delta^{k}=\sum_{k \geq 0} a_{k}^{x} \underbrace{\left(m_{k}^{x} s_{k}\right)}_{\leq C_{x}^{k}}\left(r_{k} t^{k}\right)\left(\frac{\delta}{t}\right)^{k} \leq \sum_{k \geq 0} a_{k}^{x} \underbrace{\left(r_{k} t^{k}\right)}_{\rightarrow 0, \text { as } k \rightarrow \infty}\left(\frac{\delta C_{x}}{t}\right)^{k}
$$

is absolutely convergent for each $\delta>0$. The index $x \in \Lambda$ was chosen such that $s_{k} m_{k}^{x} \leq C_{x}^{k}$ holds for all $k \in \mathbb{N}$ and it is depending on $\left(s_{k}\right)_{k} \in \mathcal{S}_{\text {Beur }}^{\mathcal{M}}$. The real number $t>0$ was chosen in such a way that $r_{k} t^{k} \rightarrow 0$ as $k \rightarrow \infty$. Hence $\left(\frac{b_{k}}{k !} r_{k} s_{k} \delta^{k}\right)_{k}$ is bounded for each $\delta>0$.

(2) $\Rightarrow(1)$ Assume that there would exist $x \in \Lambda$ such that $\sum_{k \geq 0} a_{k}^{x} t^{k}$ would have finite radius of convergence. Then there would exist $h>0$ such that $\sum_{k \geq 0}\left|a_{k}^{x}\right| n^{k}=$ $+\infty$ for each $n>h$. Put now $r_{k}:=\frac{1}{n^{k}}$ for some $n>h$ and $s_{k}:=\frac{1}{m_{k}^{x}}$.

Clearly $\left(r_{k}\right)_{k} \in \mathcal{R}_{\text {Beur,sub }}$ holds.

Also $\left(s_{k}\right)_{k}$ is as desired. By $\left(\mathcal{M}_{(\mathrm{FdB})}\right)$ for all $x \in \Lambda$ there exists $y \in \Lambda$ and $D>0$ such that for all $\alpha_{1}+\cdots+\alpha_{j}=k$ we get

$$
s_{k}:=\frac{1}{m_{k}^{x}} \leq D^{k} \frac{1}{m_{j}^{y} m_{\alpha_{1}}^{y} \cdots m_{\alpha_{j}}^{y}}=: D^{k} \hat{s}_{j} \hat{s}_{\alpha_{1}} \cdots \hat{s}_{\alpha_{j}}
$$

where we have put $\hat{s}_{j}:=\frac{1}{m_{i}^{y}}$. We have $y \leq x$, since $\left(m^{y}\right)^{\circ} \leq\left(m^{x}\right)^{\circ}$ for $y \leq x$. Clearly $\left(\hat{s}_{k}\right)_{k} \in \mathcal{S}_{\text {Beur }}^{\mathcal{M}}$, hence $\left(s_{k}\right)_{k} \in \mathcal{S}_{\text {Beur,FdB }}^{\mathcal{M}}$ and so both sequences are as considered in (2). But then there would exist $C>0$ such that for all $k \in \mathbb{N}$ :

$$
C>\frac{b_{k}}{k !} s_{k} r_{k}\left(2 n^{2}\right)^{k}=\frac{b_{k}}{k ! m_{k}^{x}} r_{k}\left(2 n^{2}\right)^{k}=a_{k}^{x} r_{k} n^{2 k} 2^{k}=a_{k}^{x} n^{k} 2^{k} .
$$

Hence $\sum_{k \geq 0}\left|a_{k}^{x}\right| n^{k} \leq C \sum_{k \geq 0} \frac{1}{2^{k}}=2 C$, a contradiction.

Using the previous result we can show: 
Proposition 4.10. Let $\mathcal{M}=\left\{M^{x}: x \in \Lambda\right\}$ be $\left(\mathcal{M}_{\mathrm{sc}}\right)$ with $\Lambda=\mathbb{R}_{>0}$ and $\left(\mathcal{M}_{(\mathrm{FdB})}\right)$. Let $E, F$ be Banach spaces, $U \subseteq E$ open and $f: U \rightarrow F$ a $\mathcal{E}$-mapping, then the following are equivalent:

(1) $f$ is $\mathcal{E}_{(\mathcal{M})}=\mathcal{E}_{(\mathcal{M})}^{\mathrm{b}}$.

(2) For each compact $K \subseteq U$, for each $\left(r_{k}\right)_{k} \in \overline{\mathcal{R}_{\text {Beur }}}$ and for each $\left(s_{k}\right)_{k} \in \mathcal{S}_{\text {Beur }}^{\mathcal{M}}$ the set

$$
\left\{\frac{f^{(k)}(a)\left(v_{1}, \ldots, v_{k}\right)}{k !} r_{k} s_{k}: a \in K, k \in \mathbb{N},\left\|v_{i}\right\|_{E} \leq 1\right\}
$$

is bounded in F.

(3) For each compact $K \subseteq U$, for each $\left(r_{k}\right)_{k} \in \mathcal{R}_{\text {Beur,sub }}$ and for each $\left(s_{k}\right)_{k} \in \mathcal{S}_{\text {Beur,FdB }}^{\mathcal{M}}$ the set

$$
\left\{\frac{f^{(k)}(a)\left(v_{1}, \ldots, v_{k}\right)}{k !} r_{k} s_{k} \delta^{k}: a \in K, k \in \mathbb{N},\left\|v_{i}\right\|_{E} \leq 1\right\}
$$

is bounded in F for each $\delta>0$.

Proof. (1) $\Rightarrow(2)$ Let $f$ be $\mathcal{E}_{(\mathcal{M})}$ and $\left(r_{k}\right)_{k},\left(s_{k}\right)_{k}$ given by (2), then we can estimate as follows (where we use Lemma5.2 below):

$$
\left\|\frac{f^{(k)}(a)}{k !} r_{k} s_{k}\right\|_{L^{k}(E, F)}=\left\|\frac{f^{(k)}(a)}{k ! m_{k}^{x} h^{k}}\right\|_{L^{k}(E, F)}|r_{k} h^{k} \underbrace{s_{k} m_{k}^{x}}_{\leq C_{x}^{k}}| \leq\left\|\frac{f^{(k)}(a)}{M_{k}^{x} h^{k}}\right\|_{L^{k}(E, F)} \underbrace{\left|r_{k}\left(C_{x} h\right)^{k}\right|}_{\rightarrow 0, \text { as } k \rightarrow \infty}
$$

for $a \in K$. We have chosen $x \in \Lambda$ depending on $\left(s_{k}\right)_{k} \in \mathcal{S}_{\text {Beur }}^{\mathcal{M}}$ such that $s_{k} m_{k}^{x} \leq C_{x}^{k}$ and $h>0$ depending on given $\left(r_{k}\right)_{k} \in \mathcal{R}_{\text {Beur }}$ such that $r_{k}\left(C_{x} h\right)^{k} \rightarrow 0$ as $k \rightarrow \infty$. (2) $\Rightarrow$ (3) Replace in (2) the sequence $\left(r_{k}\right)_{k}$ by $\left(r_{k} \delta^{k}\right)_{k}$.

(3) $\Rightarrow$ (1) Use (2) $\Rightarrow(1)$ in Lemma 4.9, Let $K \subseteq U$ be a compact set, arbitrary but fixed. Then put $b_{k}:=\sup _{a \in K}\left\|f^{(k)}(a)\right\|_{L^{k}(E, F)}$ and so for each $h>0$ and each $x \in \Lambda$ we have that $\sup _{k \in \mathbb{N}} \frac{b_{k}}{M_{x}^{k} h^{k}}<+\infty$, hence $f$ is $\mathcal{E}_{(\mathcal{M})}$.

\subsection{Beurling-case without $\left(\mathcal{M}_{(\mathrm{FdB})}\right)$}

Lemma 4.12. Let $\mathcal{M}=\left\{M^{x}: x \in \Lambda\right\}$ be $\left(\mathcal{M}_{\mathrm{sc}}\right)$ with $\Lambda=\mathbb{R}_{>0}$. For a formal power series $\sum_{k \geq 0} a_{k}^{x} t^{k}=\sum_{k \geq 0} \frac{b_{k}}{M_{k}^{x}} t^{k}, a_{k}^{x}:=\frac{b_{k}}{M_{k}^{x}}$, the following are equivalent:

(1) The series $\sum_{k \geq 0} a_{k}^{x} t^{k}$ has infinite radius of convergence for each $x \in \Lambda$.

(2) For each $\left(r_{k}\right)_{k} \in \mathcal{R}_{\text {Beur,sub }}$ and for each $\left(s_{k}\right)_{k} \in \tilde{\mathcal{S}}_{\text {Beur,sub }}^{\mathcal{M}}$ the sequence $\left(b_{k} r_{k} s_{k} \delta^{k}\right)_{k}$ is bounded for each $\delta>0$.

If $\mathcal{M}$ is $(\mathcal{M})$, then in (2) we replace $\tilde{\mathcal{S}}_{\text {Beur,sub }}^{\mathcal{M}}$ by $\tilde{\mathcal{S}}_{\text {Beur }}^{\mathcal{M}}$ 
Proof. Proceed as in Lemma 4.9, For $(2) \Rightarrow(1)$ we put $s_{k}:=\frac{1}{M_{k}^{x}}$, where $x \in \Lambda$ is the index arising by the contradiction argument.

Hence $\left(s_{k}\right)_{k} \in \tilde{\mathcal{S}}_{\text {beur,sub }}^{\mathcal{M}}$ holds whenever $\mathcal{M}$ is $\left(\mathcal{M}_{\mathrm{sc}}\right)$ since each $M^{x}$ is log-convex. If $\mathcal{M}$ is $(\mathcal{M})$, then $\left(s_{k}\right)_{k} \in \widetilde{\mathcal{S}}_{\text {Beur }}^{\mathcal{M}}$ is clear.

So we are able to prove:

Proposition 4.13. Let $\mathcal{M}=\left\{M^{x}: x \in \Lambda\right\}$ be $\left(\mathcal{M}_{\mathrm{sc}}\right)$ with $\Lambda=\mathbb{R}_{>0}$. Let $E, F$ be Banach spaces, $U \subseteq E$ open and $f: U \rightarrow F$ a $\mathcal{E}$-mapping, then the following are equivalent:

(1) $f$ is $\mathcal{E}_{(\mathcal{M})}=\mathcal{E}_{(\mathcal{M})}^{\mathrm{b}}$.

(2) For each compact $K \subseteq U$, for each $\left(r_{k}\right)_{k} \in \overline{\mathcal{R}_{\text {Beur }}}$ and for each $\left(s_{k}\right)_{k} \in \tilde{\mathcal{S}}_{\text {Beur }}^{\mathcal{M}}$ the set

$$
\left\{f^{(k)}(a)\left(v_{1}, \ldots, v_{k}\right) r_{k} s_{k}: a \in K, k \in \mathbb{N},\left\|v_{i}\right\|_{E} \leq 1\right\}
$$

is bounded in F.

(3) For each compact $K \subseteq U$, for each $\left(r_{k}\right)_{k} \in \mathcal{R}_{\text {Beur,sub }}$ and for each $\left(s_{k}\right)_{k} \in \tilde{\mathcal{S}}_{\text {Beur,sub }}^{\mathcal{M}}$ the set

$$
\left\{f^{(k)}(a)\left(v_{1}, \ldots, v_{k}\right) r_{k} s_{k} \delta^{k}: a \in K, k \in \mathbb{N},\left\|v_{i}\right\|_{E} \leq 1\right\}
$$

is bounded in F for each $\delta>0$.

If $\mathcal{M}$ is $(\mathcal{M})$, then in (3) we replace $\tilde{\mathcal{S}}_{\text {Beur,sub }}^{\mathcal{M}}$ by $\tilde{\mathcal{S}}_{\text {Beur }}^{\mathcal{M}}$

Proof. The proof is the same as for Proposition 4.10, For $(3) \Rightarrow(1)$ we use $(2) \Rightarrow(1)$ in Lemma 4.12 .

\section{Closedness under composition}

\subsection{First observations}

First we generalize [6, Lemma 4.2.]:

Lemma 5.2. Let $\mathcal{M}$ be $(\mathcal{M})$, then $\mathcal{E}_{(\mathcal{M})}=\mathcal{E}_{(\mathcal{M})}^{\mathrm{b}}$.

Proof. Let $E, F$ be convenient, $U \subseteq E$ a $c^{\infty}$-open subset and let $f: U \rightarrow F$ be a $\mathcal{E}$-mapping. Then we obtain the following equivalences, where the set $B$ runs through all closed absolutely convex bounded subsets in $E$ and $K$ runs through 
all sets in $U_{B}$ which are compact w.r.t. the norm $\|\cdot\|_{B}$ :

$$
\begin{aligned}
& f \in \mathcal{E}_{(\mathcal{M})}(U, F) \\
& \Longleftrightarrow \forall \alpha \in F^{*} \forall B \forall K \subseteq U_{B} \forall x \in \Lambda \forall h>0: \\
& \left\{\frac{(\alpha \circ f)^{(k)}(a)\left(v_{1}, \ldots, v_{k}\right)}{h^{k} M_{k}^{x}}: a \in K, k \in \mathbb{N},\left\|v_{i}\right\|_{B} \leq 1\right\} \text { is bounded in } \mathbb{R} \\
& \Longleftrightarrow \forall B \forall K \subseteq U_{B} \forall x \in \Lambda \forall h>0 \forall \alpha \in F^{*}: \\
& \alpha\left(\left\{\frac{f^{(k)}(a)\left(v_{1}, \ldots, v_{k}\right)}{h^{k} M_{k}^{x}}: a \in K, k \in \mathbb{N},\left\|v_{i}\right\|_{B} \leq 1\right\}\right) \text { is bounded in } \mathbb{R} \\
& \Longleftrightarrow \forall B \forall U^{\prime} \forall U_{B} \forall x \in \Lambda \forall h>0: \\
& \left\{\frac{f^{(k)}(a)\left(v_{1}, \ldots, v_{k}\right)}{h^{k} M_{k}^{x}}: a \in K, k \in \mathbb{N},\left\|v_{i}\right\|_{B} \leq 1\right\} \text { is bounded in } \mathbb{R} \\
& \Longleftrightarrow f \in \mathcal{E}_{(\mathcal{M})}^{\mathrm{b}}\left(U_{,} F\right) .
\end{aligned}
$$

But in general we do not have $\mathcal{E}_{\{\mathcal{M}\}}=\mathcal{E}_{\{\mathcal{M}\}}^{\mathrm{b}}$. To see this we show the following result; for the case $\mathcal{M}:=\{M\}$ see [6, Example 4.4.].

Lemma 5.3. Let $\mathcal{M}=\left\{M^{x}: x \in \Lambda\right\}$ be $\left(\mathcal{M}_{\mathrm{sc}}\right)$ with $\Lambda=\mathbb{N}_{>0}$.

Then there exists $f: \mathbb{R}^{2} \rightarrow \mathbb{R}^{\mathbb{N}_{>0}}$ which is $\mathcal{E}_{\{\mathcal{M}\}}$, but there is no reasonable topology on $\mathcal{E}_{\{\mathcal{M}\}}\left(\mathbb{R}, \mathbb{R}^{\mathbb{N}_{>0}}\right)$ such that the associated mapping $f^{\vee}: \mathbb{R} \rightarrow \mathcal{E}_{\{\mathcal{M}\}}\left(\mathbb{R}, \mathbb{R}^{\mathbb{N}_{>0}}\right)$ is $\mathcal{E}_{\{\mathcal{M}\}}^{\mathrm{b}}$.

For a "reasonable topology" on $\mathcal{E}_{\{\mathcal{M}\}}\left(\mathbb{R}, \mathbb{R}^{\mathbb{N}_{>0}}\right)$ we assume only that all pointevaluations ev $\operatorname{ev}_{\{\mathcal{M}\}}\left(\mathbb{R}, \mathbb{R}^{\mathbb{N}_{>0}}\right) \rightarrow \mathbb{R}^{\mathbb{N}_{>0}}$ are bounded linear mappings.

Proof. Consider $f: \mathbb{R}^{2} \rightarrow \mathbb{R}^{\mathbb{N}_{>0}}$ defined by $f(s, t):=\left(\theta_{x}(s t)\right)_{x \in \Lambda}$, $\theta_{x} \in \mathcal{E}_{\left\{M^{l}\right\}}^{\text {global }}(\mathbb{R}, \mathbb{R})$, see (chf), $f$ is clearly $\mathcal{E}_{\{\mathcal{M}\}}$ since each linear functional on $\mathbb{R}^{\mathbb{N}_{>0}}$ depends only on finitely many coordinates. If $f^{\vee}: \mathbb{R} \rightarrow \mathcal{E}_{\{\mathcal{M}\}}\left(\mathbb{R}, \mathbb{R}^{\mathbb{N}_{>0}}\right)$ would be $\mathcal{E}_{\{\mathcal{M}\}}^{\mathrm{b}}$, then there would exist $h>0$ and some $y \in \Lambda$ such that the set

$$
\left\{\frac{\left(f^{\vee}\right)^{(k)}(0)}{h^{k} M_{k}^{y}}: k \in \mathbb{N}\right\}
$$

would be bounded in $\mathcal{E}_{\{\mathcal{M}\}}\left(\mathbb{R}, \mathbb{R}^{\mathbb{N}_{>0}}\right)$. But if we apply the bounded linear function $\mathrm{ev}_{t}$ for $t=2 h$, then

$$
\frac{\left|\left(f^{\vee}\right)^{(k)}(0)(2 h)\right|}{h^{k} M_{k}^{y}}=\left(\frac{(2 h)^{k}\left|\theta_{x}^{(k)}(0)\right|}{h^{k} M_{k}^{y}}\right)_{x \in \Lambda} \geq\left(\frac{2^{k} M_{k}^{x}}{M_{k}^{y}}\right)_{x \in \Lambda}
$$

and so the coordinates are unbounded as $k \rightarrow \infty$ whenever $x \geq y$.

To get $\mathcal{E}_{\{\mathcal{M}\}}=\mathcal{E}_{\{\mathcal{M}\}}^{\mathrm{b}}$ we have to assume additional assumptions, see [6, Lemma 4.3.] for the constant case. 
Lemma 5.4. Let $\mathcal{M}$ be $(\mathcal{M})$, let $E, F$ be convenient and let $U \subseteq E$ be $a c^{\infty}$-open subset. Assume that there exists a Baire-vector-space-topology on the dual $F^{*}$ for which the point evaluations $\mathrm{ev}_{x}$ are continuous for all $x \in F$. Then $f: U \rightarrow F$ is $\mathcal{E}_{\{\mathcal{M}\}}$ if and only if $f$ is $\mathcal{E}_{\{\mathcal{M}\}}^{\mathrm{b}}$.

Proof. $(\Leftarrow)$ is clear.

$(\Rightarrow)$ Let $B$ a closed absolutely convex bounded subset of $E$, furthermore consider a compact set $K$ in $U_{B}$ (w.r.t. $\|\cdot\|_{B}$ ) and introduce the sets

$$
A_{x, h, C}:=\left\{\alpha \in F^{*}: \frac{\left|(\alpha \circ f)^{(k)}(a)\left(v_{1}, \ldots, v_{k}\right)\right|}{h^{k} M_{k}^{x}} \leq C, \forall k \in \mathbb{N}, a \in K,\left\|v_{i}\right\|_{B} \leq 1\right\} .
$$

These sets are closed in $F^{*}$ for the Baire-topology and $\bigcup_{x \in \Lambda, h, C>0} A_{x, h, C}=F^{*}$ holds. Then, by the Baire-property of $F^{*}$, there exist $x_{0} \in \Lambda, h_{0}, C_{0}>0$ such that the interior $\stackrel{\circ}{A}_{x_{0}, h_{0}, C_{0}}$ is non-empty. Let $\alpha_{0} \in \stackrel{\circ}{A}_{x_{0}, h_{0}, C_{0}}$, then for all $\alpha \in F^{*}$ there exists $\varepsilon>0$, such that we get $\varepsilon \alpha \in \stackrel{\circ}{A}_{x_{0}, h_{0}, C_{0}}-\alpha_{0} \Leftrightarrow \varepsilon \alpha+\alpha_{0} \in \stackrel{\circ}{A}_{x_{0}, h_{0}, C_{0}}$.

Thus for all $a \in K, k \in \mathbb{N}$ and $\left\|v_{i}\right\|_{B} \leq 1$ we get

$$
\begin{aligned}
\left|(\alpha \circ f)^{(k)}(a)\left(v_{1}, \ldots, v_{k}\right)\right| & \\
& \left.\leq \frac{1}{\varepsilon}\left(\mid\left((\varepsilon \alpha)+\alpha_{0}\right) \circ f\right)^{(k)}(a)|+|\left(\alpha_{0} \circ f\right)^{(k)}(a) \mid\right) \leq \frac{2 C_{0}}{\varepsilon} h_{0}^{k} M_{k}^{x_{0}} .
\end{aligned}
$$

So the set $\left\{\frac{f^{(k)}(a)\left(v_{1}, \ldots, v_{k}\right)}{h_{0}^{k} M_{k}^{x_{0}}}: k \in \mathbb{N}, a \in K,\left\|v_{i}\right\|_{B} \leq 1\right\}$ is weakly bounded (in $F$ ), hence bounded. Since $B$ was arbitrary we get $f \in \mathcal{E}_{\{\mathcal{M}\}}^{\mathrm{b}}$.

If the matrix is non-constant and has infinite index set, e.g. if $\mathcal{M}$ is coming from $\omega \in \mathcal{W}$ which does not have $\left(\omega_{6}\right)$ - see [9. Section 5], then another phenomenon appears.

Proposition 5.5. Let $\mathcal{M}=\left\{M^{x}: x \in \Lambda=\mathbb{N}_{>0}\right\}$ be $\left(\mathcal{M}_{\mathrm{sc}}\right)$ with $\left(\mathcal{M}_{\{\text {strict }\}}\right)$ Then there exist locally convex vector spaces $E$ and $\mathcal{E}_{\{\mathcal{M}\}}$-curves $c: \mathbb{R} \rightarrow E$ that are not $\mathcal{E}_{\left\{M^{x}\right\}}$ for any $x \in \Lambda$, i.e. $\mathcal{E}_{\{\mathcal{M}\}}(\mathbb{R}, E) \subsetneq \bigcup_{x \in \Lambda} \mathcal{E}_{\left\{M^{x}\right\}}(\mathbb{R}, E)$.

Proof. By $\left(\mathcal{M}_{\{\text {strict }\}}\right)$ we have that for each $x \in \Lambda$ we can find $x_{1} \in \Lambda, x_{1}>x$, such that $\mathcal{E}_{\left\{M^{x}\right\}} \subsetneq \mathcal{E}_{\left\{M^{x_{1}}\right\}}$. Iterating $\left(\mathcal{M}_{\{\text {strict }\}}\right)$ we obtain a strictly increasing sequence $\left(x_{i}\right)_{i \geq 0}$ with $x_{0}=x$ and $\lim _{i \rightarrow \infty} x_{i}=+\infty$, w.l.o.g. one could assume that $\mathcal{M}=\left\{M^{x_{i}}: i \in \mathbb{N}\right\}$.

So let $x \in \Lambda$ be arbitrary but from now on fixed and set $E:=\mathbb{R}^{\mathbb{N}}$. Consider a curve $c: \mathbb{R} \rightarrow \mathbb{R}^{\mathbb{N}}, c(t)=\left(c_{i}(t)\right)_{i \in \mathbb{N}}=\left(c_{0}(t), c_{1}(t), \ldots\right)$, with the following property: $c_{0}$ is $\mathcal{E}_{\left\{M^{x_{0}}\right\}}^{\mathrm{b}}$, and for each $i \geq 1$ we assume $c_{i} \in \mathcal{E}_{\left\{M^{x_{i}}\right\}} \backslash \mathcal{E}_{\left\{M^{x_{i-1}}\right\}}$.

The curve $c$ is $\mathcal{E}_{\{\mathcal{M}\}}$ since each $\alpha \in\left(\mathbb{R}^{\mathbb{N}}\right)^{*}=\mathbb{R}^{(\mathbb{N})}$ depends only on finitely many coordinates. Let $i$ be the maximal of these coordinates. Then $\alpha \circ c \in \mathcal{E}_{\left\{M^{x}{ }_{i}\right\}}(\mathbb{R}, \mathbb{R})$, thus $c \in \mathcal{E}_{\{\mathcal{M}\}}\left(\mathbb{R}, \mathbb{R}^{\mathbb{N}}\right)$.

If there would exist some $y \in \Lambda$ such that $c$ is $\mathcal{E}_{\left\{M^{y}\right\}}$, then for each $\alpha \in \mathbb{R}^{(\mathbb{N})}$ we would get that $\alpha \circ c \in \mathcal{E}_{\left\{M^{y}\right\}}(\mathbb{R}, \mathbb{R})$. According to this $y$ we choose a linear functional $\alpha$ depending on at least $i_{0}+1$ many coordinates where $x_{i_{0}}>y$. 


\subsection{Closedness under composition of $\mathcal{E}_{[\mathcal{M}]}$}

Definition 5.7. Let $E$ be a convenient vector space. $A \mathcal{E}_{[\mathcal{M}]}$-Banach-plot in $E$ is a mapping $c: D \rightarrow E$ such that $c \in \mathcal{E}_{[\mathcal{M}]}$ and $D$ denotes an open set in some Banach space $F$. It is sufficient to consider the open unit ball $D=o F$.

Using the definitions and projective representations of section 4 we can generalize [6. Theorem 4.8.].

Theorem 5.8. Let $\mathcal{M}$ be $\left(\mathcal{M}_{\mathrm{sc}}\right)$ with $\Lambda=\mathbb{R}_{>0}$, let $U \subseteq E$ be a $c^{\infty}$-open subset in a convenient vector space $E$ and $F$ be a Banach space.

If $\mathcal{M}$ has $\left(\mathcal{M}_{[\mathrm{FdB}]}\right)$ and $f: U \rightarrow F$, then $f \in \mathcal{E}_{[\mathcal{M}]}$ implies $f \circ c \in \mathcal{E}_{[\mathcal{M}]}$ for all $\mathcal{E}_{[\mathcal{M}]}$-Banach plots $\mathrm{C}$.

The converse implication holds always by the definitions given in 3.3 .

Proof. We follow the proof of [6, Theorem 4.8.] and apply Proposition 4.4 for the Roumieu- and Proposition 4.10 for the Beurling-case.

(a) Beurling-case $\mathcal{E}_{(\mathcal{M})}$.

We have to show that $f \circ c$ is $\mathcal{E}_{(\mathcal{M})}$ for each $\mathcal{E}_{(\mathcal{M})}$-Banach-plot $c: G \supseteq D \rightarrow E$, where $D$ denotes the open unit ball in an arbitrary Banach-space $G$. By (3) in Proposition 4.10 we have to prove that for each compact $K \subseteq D$ and for each $\left(r_{k}\right)_{k} \in \mathcal{R}_{\text {Beur,sub }},\left(s_{k}\right)_{k} \in \mathcal{S}_{\text {Beur,FdB }}^{\mathcal{M}}$ the set

$$
\left\{\frac{(f \circ c)^{(k)}(a)\left(v_{1}, \ldots, v_{k}\right)}{k !} r_{k} s_{k} \delta^{k}: a \in K, k \in \mathbb{N},\left\|v_{i}\right\|_{E} \leq 1\right\}
$$

is bounded in $F$ for each $\delta>0$. So let $\delta>0$, the sequences $\left(r_{k}\right)_{k},\left(s_{k}\right)_{k}$, and finally a compact (w.l.o.g. convex) set $K \subseteq D$ be given, arbitrary but from now on fixed. Then for each $\alpha \in E^{*}$ by assumption and by (2) in Proposition 4.10 applied to the sequence $\left(r_{k}(2 D \delta)^{k}\right)_{k}$ and $\left(\hat{s}_{k}\right)_{k} \in \mathcal{S}_{\text {Beur }}^{\mathcal{M}}$, where the constant $D$ is coming from $s_{k} \leq D^{k}\left(\hat{s}_{o}\right)_{k}$ (since $\left(s_{k}\right)_{k} \in \mathcal{S}_{\text {Beur,FdB }}^{\mathcal{M}}$, the set

$$
\left\{\frac{(\alpha \circ c)^{(k)}(a)\left(v_{1}, \ldots, v_{k}\right) r_{k} \hat{s}_{k}(2 D \delta)^{k}}{k !}: a \in K, k \in \mathbb{N},\left\|v_{i}\right\|_{G} \leq 1\right\}
$$

is bounded in $\mathbb{R}$. So the set

$$
\left\{\frac{c^{(k)}(a)\left(v_{1}, \ldots, v_{k}\right) r_{k} \hat{s}_{k}(2 D \delta)^{k}}{k !}: a \in K, k \in \mathbb{N},\left\|v_{i}\right\|_{G} \leq 1\right\}
$$

is contained in some closed absolutely convex bounded subset $B$ of $E$, hence

$$
\frac{\left\|c^{(k)}(a)\right\|_{L^{k}\left(G, E_{B}\right)} r_{k} \hat{s}_{k} \delta^{k}}{k !} \leq \frac{1}{(2 D)^{k}}
$$

We proceed now as in [6, Theorem 4.8.]. $c(K)$ is compact in $E_{B}$ since the mapping $c: K \rightarrow E_{B}$ is Lipschitzian: For all $a, b \in K$ we get $c(a)-c(b) \in \frac{\|a-b\|_{G}}{2 D r_{1} \hat{s}_{1} \delta} B$. Then 
we estimate for all $\delta>0$ and $k \in \mathbb{N}_{>0}$ as follows:

$$
\begin{aligned}
& \left\|\frac{(f \circ c)^{(k)}(a)}{k !} r_{k} s_{k} \delta^{k}\right\|_{L^{k}(G, F)} \\
& \leq \sum_{j \geq 0} \sum_{\alpha \in \mathbb{N}_{>0}^{j}, \Sigma_{i=1}^{j} \alpha_{i}=k} D^{k} \frac{\left\|f^{(j)}(c(a))\right\|_{L^{j}\left(E_{B}, F\right)}}{j !} \underbrace{\prod_{i=1}^{j} \frac{\left\|c^{\left(\alpha_{i}\right)}(a)\right\|_{L^{\alpha_{i}}\left(G, E_{B}\right)^{k}} r_{\alpha_{i}} \hat{s}_{\alpha_{i}} \delta^{\alpha_{i}}}{\alpha_{i} !}}_{\leq \frac{1}{(2 D)^{\alpha_{1}} \cdots \frac{1}{(2 D)^{\alpha_{j}}}=\frac{1}{(2 D)^{k}}}} \\
& \leq\left(\frac{1}{2}\right)^{k} \sum_{j \geq 0} \sum_{\alpha \in \mathbb{N}_{>0}^{j}, \Sigma_{i=1}^{j} \alpha_{i}=k} \underbrace{\frac{\left\|f^{(j)}(c(a))\right\|_{L^{j}\left(E_{B}, F\right)}}{j ! m_{j}^{x}}}_{(\star) \leq C h^{j}} \underbrace{\left(\hat{s}_{j} m_{j}^{x}\right)}_{\leq C_{1}^{j}} \\
& \leq\left(C C_{1}\right)\left(\frac{1}{2}\right)^{k} \cdot \sum_{j \geq 0}\left(\begin{array}{l}
k-1 \\
j-1
\end{array}\right)\left(h C_{1}\right)^{j-1}=\left(C h C_{1}\right)\left(\frac{1}{2}\right)^{k}\left(1+C_{1} h\right)^{k-1} \\
& \leq\left(C_{1} C_{1}\right)\left(\frac{\left(1+C_{1} h\right)}{2}\right)^{k} \text {. }
\end{aligned}
$$

We have to choose $x \in \Lambda$ according to $\left(\hat{s}_{j}\right)_{j} \in \mathcal{S}_{\text {Beur }}^{\mathcal{M}}$ (arising in $\mathcal{S}_{\text {Beur,FdB }}^{\mathcal{M}}$ ) such that $\hat{s}_{j} m_{j}^{x} \leq C_{1}^{j}$ for some constant $C_{1}>0$ and all $j \in \mathbb{N}$. Since $f \in \mathcal{E}_{(\mathcal{M})}$, we obtain the estimate $(\star)$ with this index $x$ and arbitrary $h>0$ for a constant $C=C_{x, h}$ and all $j \in \mathbb{N}$. Finally we can choose $h:=\frac{1}{C_{1}}$ and so the expression at the beginning is bounded by $C=C_{x, 1 / C_{1}}$.

(b) Roumieu-case $\mathcal{E}_{\{\mathcal{M}\}}$.

Use Proposition 4.4 and by (3) there it is sufficient to show that each compact $K \subseteq D$ and for each $\left(r_{k}\right)_{k} \in \mathcal{R}_{\text {Roum,sub }},\left(s_{k}\right)_{k} \in \mathcal{S}_{\text {Roum,FdB }}^{\mathcal{M}}$ there exists $\varepsilon>0$ such that the set

$$
\left\{\frac{(f \circ c)^{(k)}(a)\left(v_{1}, \ldots, v_{k}\right)}{k !} r_{k} s_{k} \varepsilon^{k}: a \in K, k \in \mathbb{N},\left\|v_{i}\right\|_{E} \leq 1\right\}
$$

is bounded in $F$.

We use the same proof as above and replace in (2) in Proposition 4.4 the sequence $\left(r_{k}\right)_{k}$ by $\left((2 D)^{k} r_{k}\right)_{k}$, where $D$ is the constant arising in $s_{k} \leq D^{k}\left(\hat{s}_{o}\right)_{k}$ (since $\left(s_{k}\right)_{k} \in$ $\mathcal{S}_{\text {Roum,FdB }}^{\mathcal{M}}$ and so $\left(\hat{s}_{k}\right)_{k} \in \mathcal{S}_{\text {Roum }}^{\mathcal{M}}$. Then we take $\delta=1$ in (5.1), in (5.2) and in the Lipschitz-argument. We can use now precisely the same estimate as for the Beurling-case (for $\delta=1$ ) and so we have shown (5.3) for $\varepsilon=\frac{2}{\left(1+C_{1} h\right)}$. Note that $f \in \mathcal{E}_{\{\mathcal{M}\}}$, hence we have to consider $x \in \Lambda$ and $h>0$ sufficiently large to obtain estimate $(\star)$ for some constant $C$. According to this chosen $x \in \Lambda$ we can estimate $\hat{s}_{j} m_{j}^{x} \leq C_{1}^{j}$ for a constant $C_{1}$ and all $j \in \mathbb{N}$, since $\left(\hat{s}_{j}\right)_{j} \in \mathcal{S}_{\text {Roum }}^{\mathcal{M}}$.

Using Theorem 5.8 we can generalize [6, Theorem 4.9.]. 
Theorem 5.9. Let $\mathcal{M}$ be $\left(\mathcal{M}_{\mathrm{sc}}\right)$ with $\Lambda=\mathbb{R}_{>0}$. Let $E, F, G$ be convenient vector spaces, $U \subseteq E$ and $V \subseteq F$ be $c^{\infty}$-open and $f: U \rightarrow F, g: V \rightarrow G$ with $f(U) \subseteq V$.

(a) If $\mathcal{M}_{(\mathrm{FdB})}$ then $f, g \in \mathcal{E}_{(\mathcal{M})}$ implies $g \circ f \in \mathcal{E}_{(\mathcal{M})}$.

(b) If $\mathcal{M}_{\{\mathrm{FdB}\}}$, then $f, g \in \mathcal{E}_{\{\mathcal{M}\}}$ implies $g \circ f \in \mathcal{E}_{\{\mathcal{M}\}}$.

Proof. By definition of $\mathcal{E}_{[\mathcal{M}]}$ we have to show that for all closed absolutely convex bounded subsets $B \subseteq E$ and for all $\alpha \in G^{*}$ the composite $\alpha \circ g \circ f \circ i_{B}: U_{B} \rightarrow \mathbb{R}$ is $\mathcal{E}_{[\mathcal{M}]}$. By assumption $f \circ i_{B} \in \mathcal{E}_{[\mathcal{M}]}$ and $\alpha \circ g \in \mathcal{E}_{[\mathcal{M}]}$ hold, so we can use Theorem 5.8 to obtain the desired implication. Note that $f \circ i_{B}$ is a $\mathcal{E}_{[\mathcal{M}]}$-Banach plot.

\section{Exponential laws for $\mathcal{E}_{[\mathcal{M}]}$}

We start with the generalization of [6, Lemma 5.1.].

Lemma 6.1. Let $\mathcal{M}$ be $(\mathcal{M})$ or $\left(\mathcal{M}_{\mathrm{sc}}\right)$ with $\Lambda=\mathbb{R}_{>0}$, let $E$ be Banach and $U \subseteq E$ open. Let $F$ be convenient and $\mathcal{B}$ a family of bounded linear functionals on $F$ which together detect bounded sets, i.e. $B \subseteq E$ is bounded in $E$ if and only if $\alpha(B)$ is bounded in $\mathbb{R}$ for all $\alpha \in \mathcal{B}$. Then we have

$$
f \in \mathcal{E}_{[\mathcal{M}]}(U, F) \Leftrightarrow \alpha \circ f \in \mathcal{E}_{[\mathcal{M}]}(U, \mathbb{R}) \quad \forall \alpha \in \mathcal{B} .
$$

Proof. For $\mathcal{E}$-curves this follows by [4, 2.1., 2.11.], and so by composing with such curves for $\mathcal{E}$-mappings $f: U \rightarrow F$.

In the Roumieu-case we use $(1) \Leftrightarrow(2)$ in Proposition 4.7. Hence for arbitrary $\alpha \in F^{*}$ the mapping $\alpha \circ f$ is $\mathcal{E}_{\{\mathcal{M}\}}$ if and only if for each compact $K \subseteq U$ the set

$$
\left\{(\alpha \circ f)^{(k)}(a)\left(v_{1}, \ldots, v_{k}\right) r_{k} s_{k}: a \in K, k \in \mathbb{N},\left\|v_{i}\right\|_{E} \leq 1\right\}
$$

is bounded in $\mathbb{R}$ for each $\left(r_{k}\right)_{k} \in \mathcal{R}_{\text {Roum }}$ and for each $\left(s_{k}\right)_{k} \in \tilde{\mathcal{S}}_{\text {Roum }}^{\mathcal{M}}$ So the smooth mapping $f: U \rightarrow F$ is $\mathcal{E}_{\{\mathcal{M}\}}$ if and only if the set

$$
\left\{f^{(k)}(a)\left(v_{1}, \ldots, v_{k}\right) r_{k} s_{k}: a \in K, k \in \mathbb{N},\left\|v_{i}\right\|_{E} \leq 1\right\}
$$

is bounded in $F$, for each compact $K \subseteq U,\left(r_{k}\right)_{k} \in \mathcal{R}_{\text {Roum }}$ and for each $\left(s_{k}\right)_{k} \in \tilde{\mathcal{S}}_{\text {Roum }}^{\mathcal{M}}$

Because $\mathcal{B}$ detects bounded sets we can replace in the above equivalences $F^{*}$ by $\mathcal{B}$.

For the Beurling-case proceed analogously and use $(1) \Leftrightarrow(2)$ in Proposition 4.13 ,

Now we are able to prove Cartesian closedness for classes $\mathcal{E}_{[\mathcal{M}]}$ and so generalize [6. Theorem 5.2.].

Theorem 6.2. Let $\mathcal{M}$ be $\left(\mathcal{M}_{\mathrm{sc}}\right)$ with $\Lambda=\mathbb{R}_{>0}$, let $U_{i} \subseteq E_{i}$ be $c^{\infty}$-open subsets in convenient vector spaces $E_{i}$ for $i=1,2$ and moreover let $F$ be also a convenient vector space. Then we obtain: 
(a) If $\left(\mathcal{M}_{\{\mathrm{mg}\}}\right)$, then

$$
f \in \mathcal{E}_{\{\mathcal{M}\}}\left(U_{1} \times U_{2}, F\right) \Longleftrightarrow f^{\vee} \in \mathcal{E}_{\{\mathcal{M}\}}\left(U_{1}, \mathcal{E}_{\{\mathcal{M}\}}\left(U_{2}, F\right)\right) .
$$

(b) If $\left(\mathcal{M}_{(\mathrm{mg})}\right)$, then

$$
f \in \mathcal{E}_{(\mathcal{M})}\left(U_{1} \times U_{2}, F\right) \Longleftrightarrow f^{\vee} \in \mathcal{E}_{(\mathcal{M})}\left(U_{1}, \mathcal{E}_{(\mathcal{M})}\left(U_{2}, F\right)\right) .
$$

Important remarks:

(i) In both cases $(\Longleftarrow)$ holds also without $\left(\mathcal{M}_{\{\mathrm{mg}\}}\right)$ respectively $\left(\mathcal{M}_{(\mathrm{mg})}\right)$.

(ii) To prove $(\Longleftarrow)$ it is sufficient to assume that $\mathcal{M}$ is $(\mathcal{M})$ and $\left(\mathcal{M}_{[\mathrm{alg}]}\right)$.

(iii) For the proof it is not necessary to assume that $\mathcal{E}_{\{\mathcal{M}\}}$ respectively $\mathcal{E}_{(\mathcal{M})}$ is a category, i.e. closedness under composition.

(iv) If $\mathcal{M}$ is $\left(\mathcal{M}_{\mathrm{sc}}\right)$ with $\Lambda=\mathbb{R}_{>0},\left(\mathcal{M}_{[\mathrm{mg}]}\right)$ and $\left(\mathcal{M}_{[\mathrm{FdB}]}\right)$, then by Theorem 6.2 and Theorem 5.9 the category $\mathcal{E}_{[\mathcal{M}]}$ is cartesian closed.

Proof. The technique and methods are completely analogous to [6, Theorem 5.2.], for convenience of the reader we give the full proof.

As shown in [4, 3.12.] we have $\mathcal{E}\left(U_{1} \times U_{2}, F\right) \cong \mathcal{E}\left(U_{1}, \mathcal{E}\left(U_{2}, F\right)\right)$. So we assume form now on that all occurring mappings are smooth. Let $B \subseteq E_{1} \times E_{2}$ and $B_{i} \subseteq E_{i}, i=1,2$, where $B, B_{1}, B_{2}$ run through all closed absolutely convex bounded subsets. Similarly as shown in [6, Theorem 5.2.] we get:

$$
\begin{aligned}
f & \in \mathcal{E}_{[\mathcal{M}]}\left(U_{1} \times U_{2}, F\right) \\
& \Leftrightarrow \forall \alpha \in F^{*} \forall B: \alpha \circ f \circ i_{B} \in \mathcal{E}_{[\mathcal{M}]}\left(\left(U_{1} \times U_{2}\right)_{B}, \mathbb{R}\right) \\
& \Leftrightarrow \forall \alpha \in F^{*} \forall B_{1}, B_{2}: \alpha \circ f \circ\left(i_{B_{1}} \times i_{B_{2}}\right) \in \mathcal{E}_{[\mathcal{M}]}\left(\left(U_{1}\right)_{B_{1}} \times\left(U_{2}\right)_{B_{2}}, \mathbb{R}\right)
\end{aligned}
$$

and

$$
\begin{aligned}
& f^{\vee} \in \mathcal{E}_{[\mathcal{M}]}\left(U_{1}, \mathcal{E}_{[\mathcal{M}]}\left(U_{2}, F\right)\right) \\
& \Leftrightarrow \forall B_{1}: f^{\vee} \circ i_{B_{1}} \in \mathcal{E}_{[\mathcal{M}]}\left(\left(U_{1}\right)_{B_{1}}, \mathcal{E}_{[\mathcal{M}]}\left(U_{2}, F\right)\right) \\
& \Leftrightarrow \forall \alpha \in F^{*} \forall B_{1}, B_{2}: \mathcal{E}_{[\mathcal{M}]}\left(i_{B_{2}}, \alpha\right) \circ f^{\vee} \circ i_{B_{1}} \in \mathcal{E}_{[\mathcal{M}]}\left(\left(U_{1}\right)_{B_{1}}, \mathcal{E}_{[\mathcal{M}]}\left(\left(U_{2}\right)_{B_{2}}, \mathbb{R}\right)\right),
\end{aligned}
$$

where Lemma 6.1 is used and note that the linear mappings $\mathcal{E}_{[\mathcal{M}]}\left(i_{B_{2}}, \alpha\right)$ generate the bornology.

With these preparations we are able to restrict ourselves to $U_{i} \subseteq E_{i}$ open sets in Banach spaces $E_{i}$ and $F=\mathbb{R}$. We start now with $(\Longrightarrow)$ for both cases.

Let $f \in \mathcal{E}_{[\mathcal{M}]}\left(U_{1} \times U_{2}, \mathbb{R}\right)$, then clearly $f^{\vee}$ takes values in the space $\mathcal{E}_{[\mathcal{M}]}\left(U_{2}, \mathbb{R}\right)$. First we show that Claim. $f^{\vee}: U_{1} \rightarrow \mathcal{E}_{[\mathcal{M}]}\left(U_{2}, \mathbb{R}\right)$ is $\mathcal{E}$ with $d^{j} f^{\vee}=\left(\partial_{1}^{j} f\right)^{\vee}$. $\mathcal{E}_{[\mathcal{M}]}\left(U_{2}, \mathbb{R}\right)$ are convenient vector spaces, hence by [4, 5.20.] it suffices to prove that the iterated unidirectional derivatives $d_{v}^{j} f^{\vee}(x)$ exist, are equal to $\partial_{1}^{j} f(x, \cdot)\left(v^{j}\right)$, and are separately bounded for $x$ and $v$ in compact subsets. For $j=1$ and $x, v, y$ 
fixed we consider the smooth curve $c: t \mapsto f(x+t v, y)$. Then, by the fundamental theorem of calculus, we obtain:

$$
\begin{aligned}
& \frac{f^{\vee}(x+t v)-f^{\vee}(x)}{t}(y)-\left(\partial_{1} f\right)^{\vee}(x)(y)(v)=\frac{c(t)-c(0)}{t}-c^{\prime}(0) \\
& =t \int_{0}^{1} s \int_{0}^{1} c^{\prime \prime}(t s r) d r d s=t \int_{0}^{1} s \int_{0}^{1} \partial_{1}^{2} f(x+t s r v, y)(v, v) d r d s .
\end{aligned}
$$

$\left(\partial_{1}^{2} f\right)^{\vee}\left(K_{1}\right)\left(o\left(E_{1} \times E_{1}\right)\right)$ is bounded in $\mathcal{E}_{[\mathcal{M}]}\left(U_{2}, \mathbb{R}\right)$ and for each compact set $K_{1} \subseteq U_{1}$ this expression is Mackey-convergent to 0 in $\mathcal{E}_{[\mathcal{M}]}\left(U_{2}, \mathbb{R}\right)$ as $t \rightarrow 0$. Hence $d_{v} f^{\vee}(x)$ exists an is equal to $\partial_{1} f(x, \cdot)(v)$.

The induction argument is completely the same as in [6, Theorem 5.2.].

We distinguish now between the Roumieu- and the Beurling-case.

The Beurling-case.

We have to show that $f^{\vee}: U_{1} \rightarrow \mathcal{E}_{(\mathcal{M})}\left(U_{2}, \mathbb{R}\right)$ is $\mathcal{E}_{(\mathcal{M})}$.

By Lemma 6.1 it suffices to prove that $f^{\vee}: U_{1} \rightarrow \mathcal{E}_{M^{x}, h}\left(E_{2} \supseteq K_{2}, \mathbb{R}\right)$ is $\mathcal{E}_{(\mathcal{M})}^{\mathrm{b}}=\mathcal{E}_{(\mathcal{M})}$ for each $K_{2} \subseteq U_{2}$ compact, each $h>0$ and $x \in \Lambda=\mathbb{R}_{>0}$. This holds, because each $\alpha \in\left(\mathcal{E}_{(\mathcal{M})}\left(U_{2}, \mathbb{R}\right)\right)^{*}$ factorizes over $\mathcal{E}_{M^{x}, h}\left(E_{2} \supseteq K_{2}, \mathbb{R}\right)$ for some $K_{2}, h$ and $x$.

So we have to show that for each compact sets $K_{1} \subseteq U_{1}, K_{2} \subseteq U_{2}$, each $h_{1}, h_{2}>0$ and each $x_{1}, x_{2} \in \Lambda$, the set

$$
\left\{\frac{d^{k_{1}} f^{\vee}\left(a_{1}\right)\left(v_{1}^{1}, \ldots, v_{k_{1}}^{1}\right)}{h_{1}^{k_{1}} M_{k_{1}}^{x_{1}}}: a_{1} \in K_{1}, k_{1} \in \mathbb{N},\left\|v_{j}^{1}\right\|_{E_{1}} \leq 1\right\}
$$

is bounded in the space $\mathcal{E}_{M^{x_{2}, h_{2}}}\left(E_{2} \supseteq K_{2}, \mathbb{R}\right)$. Equivalently, for all compact sets $K_{1}, K_{2}$, for all $h_{1}, h_{1}>0$ and all $x_{1}, x_{2} \in \Lambda$ the set

$$
\left\{\frac{\partial_{2}^{k_{2}} \partial_{1}^{k_{1}} f\left(a_{1}, a_{2}\right)\left(v_{1}^{1}, \ldots, v_{k_{1}}^{1} ; v_{1}^{2}, \ldots, v_{k_{2}}^{2}\right)}{h_{2}^{k_{2}} h_{1}^{k_{1}} M_{k_{2}}^{x_{2}} M_{k_{1}}^{x_{1}}}: a_{i} \in K_{i}, k_{i} \in \mathbb{N},\left\|v_{j}^{i}\right\|_{E_{i}} \leq 1 ; i=1,2\right\}
$$

is bounded in $\mathbb{R}$. 
Let $a_{1} \in K_{1}, k_{1} \in \mathbb{N}$, then we obtain the following estimate:

$$
\begin{aligned}
& \left\|\frac{d^{k_{1}} f^{\vee}\left(a_{1}\right)\left(v_{1}^{1}, \ldots, v_{k_{1}}^{1}\right)}{h_{1}^{k_{1}} M_{k_{1}}^{x_{1}}}\right\|_{M^{x_{2}, K_{2}, h_{2}}}^{J} \\
& =\sup \left\{\frac{\left|\partial_{2}^{k_{2}} \partial_{1}^{k_{1}} f\left(a_{1}, a_{2}\right)\left(v_{1}^{1}, \ldots, v_{k_{1}}^{1} ; v_{1}^{2}, \ldots, v_{k_{2}}^{2}\right)\right|}{h_{1}^{k_{1}} h_{2}^{k_{2}} M_{k_{1}}^{x_{1}} M_{k_{2}}^{x_{2}}}: a_{2} \in K_{2}, k_{2} \in \mathbb{N},\left\|v_{j}^{2}\right\|_{E_{2}} \leq 1\right\} \\
& \underbrace{\leq}_{\left(\mathcal{M}_{(\mathrm{mg})}\right)} \sup \left\{C^{k_{1}+k_{2}} \frac{\left|\partial_{2}^{k_{2}} \partial_{1}^{k_{1}} f\left(a_{1}, a_{2}\right)\left(v_{1}^{1}, \ldots, v_{k_{1}}^{1} ; v_{1}^{2}, \ldots, v_{k_{2}}^{2}\right)\right|}{h_{1}^{k_{1}} h_{2}^{k_{2}} M_{k_{1}+k_{2}}^{y}}:\right. \\
& \leq \sup \left\{\frac{\left|\partial_{2}^{k_{2}} \partial_{1}^{k_{1}} f\left(a_{1}, a_{2}\right)\left(v_{1}^{1}, \ldots, v_{k_{1}}^{1} ; v_{1}^{2}, \ldots, v_{k_{2}}^{2}\right)\right|}{h^{k_{1}+k_{2} M_{k_{1}+k_{2}}^{y}}:}:\right.
\end{aligned}
$$

where we have put $h:=\frac{1}{C} \min \left\{h_{1}, h_{2}\right\}$. Note that $f$ is $\mathcal{E}_{(\mathcal{M})}$ and so for arbitrary $h_{1}, h_{2}>0$ and $x_{1}, x_{2} \in \Lambda$ we can find $y \in \Lambda$ and $h>0$ such that the last inequality is valid. This shows that $f^{\vee}$ is $\mathcal{E}_{(\mathcal{M})}$.

The Roumieu-case.

By Lemma 6.1 it suffices to prove that $f^{\vee}: U_{1} \rightarrow \underset{x_{2} \in \Lambda}{\lim } \underset{h_{2}>0}{\lim } \mathcal{E}_{M^{x_{2}, h_{2}}}\left(E_{2} \supseteq K_{2}, \mathbb{R}\right)$ is $\mathcal{E}_{\{\mathcal{M}\}}^{\mathrm{b}} \subseteq \mathcal{E}_{\{\mathcal{M}\}}$ for each compact set $K_{2} \subseteq U_{2}$. This holds because each $\alpha \in\left(\mathcal{E}_{\{\mathcal{M}\}}\left(U_{2}, \mathbb{R}\right)\right)^{*}$ factorizes over some $\underset{x_{2} \in \Lambda \text { lim }}{\lim } \mathcal{E}_{h_{2}>0} \mathcal{E}_{M^{x_{2}, h_{2}}}\left(E_{2} \supseteq K_{2}, \mathbb{R}\right)$.

So we have to prove that for all $K_{1} \subseteq U_{1}, K_{2} \subseteq U_{2}$ compact there exist $h_{1}>0$ and

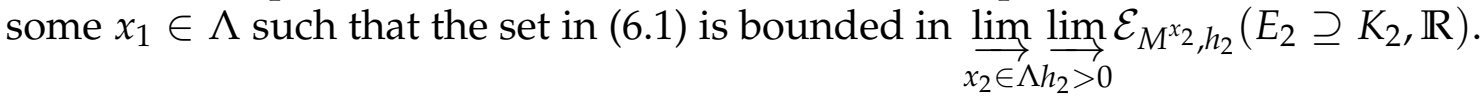

Equivalently, we have to show that for all $K_{1}, K_{2}$ compact there exist $h_{1}, h_{2}>0$ and $x_{1}, x_{2} \in \Lambda$ such that the set in (6.2) is bounded in $\mathbb{R}$.

We can use now the same estimate as for the above Beurling-case and use $\left(\mathcal{M}_{\{\mathrm{mg}\}}\right)$. First, because $f$ is $\mathcal{E}_{\{\mathcal{M}\}}$ and by (3) in Proposition 3.4 we obtain that there exist some $h>0$ and $y \in \Lambda$, such that the last set

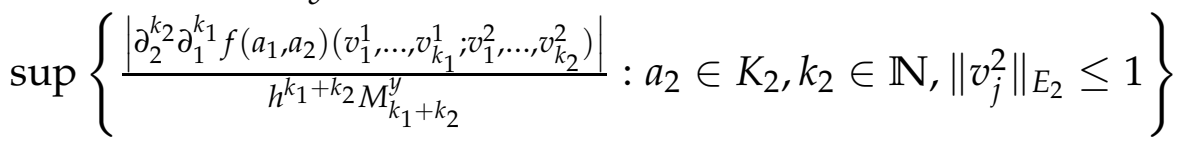

in the Beurling estimate is bounded. For this $y \in \Lambda$ we obtain by $\left(\mathcal{M}_{\{\mathrm{mg}\}}\right)$ that there exist some $x_{1}, x_{2} \in \Lambda$ and $C>0$ such that $M_{j+k}^{y} \leq C^{j+k} M_{j}^{x_{1}} M_{k}^{x_{2}}$ holds for all $j, k \in \mathbb{N}$. So we can put in the estimate now $h_{i}:=C h$ for $i=1,2$ to get, that $f^{\vee}$ is $\mathcal{E}_{\{\mathcal{M}\}}$.

Now we start with $(\Longleftarrow)$ for both cases. 
Let $f^{\vee}: U_{1} \rightarrow \mathcal{E}_{[\mathcal{M}]}\left(U_{2}, \mathbb{R}\right)$ be $\mathcal{E}_{[\mathcal{M}]}$. The mapping $f^{\vee}: U_{1} \rightarrow \mathcal{E}_{[\mathcal{M}]}\left(U_{2}, \mathbb{R}\right) \rightarrow$ $\mathcal{E}\left(U_{2}, \mathbb{R}\right)$ is $\mathcal{E}$, hence it remains to show that $f \in \mathcal{E}_{[\mathcal{M}]}\left(U_{1} \times U_{2}, \mathbb{R}\right)$.

The Beurling-case.

For each compact $K_{2} \subseteq U_{2}$, each $h_{2}>0$ and each $x_{2} \in \Lambda$, the mapping $f^{\vee}: U_{1} \rightarrow \mathcal{E}_{M^{x_{2}, h_{2}}}\left(E_{2} \supseteq K_{2}, \mathbb{R}\right)$ is $\mathcal{E}_{(\mathcal{M})}^{\mathrm{b}}=\mathcal{E}_{(\mathcal{M})}$. This means that for all compact $K_{1} \subseteq U_{1}, K_{2} \subseteq U_{2}$, each $h_{1}, h_{2}>0$ and each $x_{1}, x_{2} \in \Lambda$ the set in (6.1) is bounded in $\mathcal{E}_{M^{x_{2}, h_{2}}}\left(E_{2} \supseteq K_{2}, \mathbb{R}\right)$. Because it is contained in the space $\mathcal{E}_{M^{x_{2}, K_{2}, h_{2}}}\left(U_{2}, \mathbb{R}\right):=$ $\left\{f \in \mathcal{E}\left(U_{2}, \mathbb{R}\right):\left.j^{\infty}(f)\right|_{K_{2}} \in \mathcal{E}_{M^{x_{2}, h_{2}}}\left(E_{2} \supseteq K_{2}, \mathbb{R}\right)\right\}$ with semi-norm $\|f\|_{M^{x_{2}, K_{2}, h_{2}}}^{J}:=$ $\left\|\left.j^{\infty}(f)\right|_{K_{2}}\right\|_{M^{x_{2}, h_{2}}}^{J}$ it is also bounded in this space and so the set in (6.2) is bounded in $\mathbb{R}$.

By assumption each $M^{x}$ is log-convex and so $M_{j}^{x} M_{k}^{x} \leq M_{j+k}^{x}$ for all $j, k \in \mathbb{N}$. For the next estimate $\left(\mathcal{M}_{(\text {alg })}\right)$ would be sufficient. Let $a_{1} \in K, k_{1} \in \mathbb{N}$ and $\left\|v_{j}^{1}\right\|_{E_{1}} \leq 1$, then:

$$
\begin{aligned}
&+\infty>\left\|\frac{d^{k_{1}} f^{\vee}\left(a_{1}\right)\left(v_{1}^{1}, \ldots, v_{k_{1}}^{1}\right)}{h_{1}^{k_{1}} M_{k_{1}}^{x_{1}}}\right\|_{M^{x_{2}, K_{2}, h_{2}}}^{J} \\
&=\sup \left\{\frac{\left|\partial_{2}^{k_{2}} \partial_{1}^{k_{1}} f\left(a_{1}, a_{2}\right)\left(v_{1}^{1}, \ldots, v_{k_{1}}^{1} ; v_{1}^{2}, \ldots, v_{k_{2}}^{2}\right)\right|}{h_{1}^{k_{1}} h_{2}^{k_{2}} \cdot M_{k_{1}}^{x_{1}} M_{k_{2}}^{x_{2}}}:\right. \\
& \geq \sup \left\{\frac{\left.\mid \partial_{2} \in K_{2}, k_{2} \in \mathbb{N},\left\|v_{j}^{2}\right\|_{E_{2}} \leq 1\right\}}{h^{k_{1}}{ }^{k_{1}} f\left(a_{1}, M_{k_{1}}\right)\left(v_{1}^{1}, \ldots, v_{k_{1}}^{1} ; v_{1}^{2}, \ldots, v_{k_{2}}^{2}\right) \mid}:\right. \\
&\left.a_{2} \in K_{2}, k_{2} \in \mathbb{N},\left\|v_{j}^{2}\right\|_{E_{2}} \leq 1\right\}
\end{aligned}
$$

where we have put $y:=\max \left\{x_{1}, x_{2}\right\}$ and $h:=\max \left\{h_{1}, h_{2}\right\}$ (put $h:=$ $C \max \left\{h_{1}, h_{2}\right\}$, where $y \in \Lambda$ and $C>0$ are coming from $\left.\left(\mathcal{M}_{(\mathrm{alg})}\right)\right)$. So we have shown that $f$ is $\mathcal{E}_{(\mathcal{M})}$.

The Roumieu-case.

For each compact $K_{2} \subseteq U_{2}$ the mapping $f^{\vee}: U_{1} \rightarrow \underset{x_{2} \in \Lambda}{\lim } \underset{h_{2}>0}{\lim } \mathcal{E}_{M^{x_{2}}, h_{2}}\left(E_{2} \supseteq K_{2}, \mathbb{R}\right)$ is $\mathcal{E}_{\{\mathcal{M}\}}$. By (3) in Proposition 3.4 the dual space $\left(\underset{x_{2} \in \Lambda}{\lim _{h_{2}>0}} \underset{\lim _{M^{2}}}{\mathcal{E}_{M^{2}}} \mathcal{E}_{h_{2}}\left(E_{2} \supseteq K_{2}, \mathbb{R}\right)\right)^{*}$ can be equipped with the Baire-vector-space-topology of the countable limit of Banach spaces $\lim _{x_{2} \in \Lambda} \lim _{h_{2}>0}\left(\mathcal{E}_{M^{x_{2}, h_{2}}}\left(E_{2} \supseteq K_{2}, \mathbb{R}\right)\right)^{*}$.

Now we can use Lemma 5.4 to conclude that the mapping $f^{\vee}: U_{1} \rightarrow \underset{x_{2} \in \Lambda}{\lim _{h_{2}>0}}$ $\mathcal{E}_{M^{x_{2}, h_{2}}}\left(E_{2} \supseteq K_{2}, \mathbb{R}\right)$ is $\mathcal{E}_{\{\mathcal{M}\}}^{\mathrm{b}}$.

By (3) in Proposition 3.4 this inductive limit is countable and compactly regular and so for each compact $K_{1} \subseteq U_{1}$ there exist $h_{1}>0$ and $x_{1} \in \Lambda$ such that the set in 
(6.1) is bounded in $\mathcal{E}_{M^{x_{2}, h_{2}}}\left(E_{2} \supseteq K_{2}, \mathbb{R}\right)$ for some $h_{2}>0$ and $x_{2} \in \Lambda$. Because it is contained $\mathcal{E}_{M^{x_{2}, K_{2}, h_{2}}}\left(U_{2}, \mathbb{R}\right):=\left\{f \in \mathcal{E}\left(U_{2}, \mathbb{R}\right):\left.j^{\infty}(f)\right|_{K_{2}} \in \mathcal{E}_{M^{x_{2}, h_{2}}}\left(E_{2} \supseteq K_{2}, \mathbb{R}\right)\right\}$ with semi-norm $\|f\|_{M^{x_{2}, K_{2}, h_{2}}}^{J}:=\left\|\left.j^{\infty}(f)\right|_{K_{2}}\right\|_{M^{x_{2}, h_{2}}}^{J}$ it is also bounded in this space and so the set in (6.2) is bounded (in $\mathbb{R}$ ) with those given $h_{1}, h_{2}, x_{1}, x_{2}$.

But now we can use the same estimate as in the above Beurling-case to conclude that $f$ is $\mathcal{E}_{\{\mathcal{M}\}}$. Similarly $\left(\mathcal{M}_{\{\mathrm{alg}\}}\right)$ would be sufficient for this step.

Using Theorem 6.2 we can prove now the matrix generalization of [6, Corollary 5.5.]:

Corollary 6.3. Let $\mathcal{M}$ be a weight matrix as assumed in Theorem 6.2 Let $E, F, E_{i}, F_{i}, G$ be convenient vector spaces and let $U$ and $V$ be $c^{\infty}$-open subsets. Then we get

(1) The exponential law

$\mathcal{E}_{[\mathcal{M}]}\left(U, \mathcal{E}_{[\mathcal{M}]}(V, G)\right) \cong \mathcal{E}_{[\mathcal{M}]}(U \times V, G)$

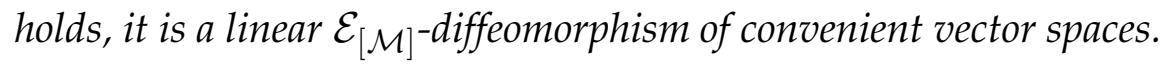

The following mappings are $\mathcal{E}_{[\mathcal{M}]}$ :

(2) ev : $\mathcal{E}_{[\mathcal{M}]}(U, F) \times U \rightarrow F$ given by ev $(f, x)=f(x)$.

(3) ins : $E \rightarrow \mathcal{E}_{[\mathcal{M}]}(F, E \times F)$ given by ins $(x)(y)=(x, y)$.

(4) $(\cdot)^{\wedge}: \mathcal{E}_{[\mathcal{M}]}\left(U, \mathcal{E}_{[\mathcal{M}]}(V, G)\right) \rightarrow \mathcal{E}_{[\mathcal{M}]}(U \times V, G)$.

(5) $(\cdot)^{\vee}: \mathcal{E}_{[\mathcal{M}]}(U \times V, G) \rightarrow \mathcal{E}_{[\mathcal{M}]}\left(U, \mathcal{E}_{[\mathcal{M}]}(V, G)\right)$.

(6) $\prod: \prod_{i} \mathcal{E}_{[\mathcal{M}]}\left(E_{i}, F_{i}\right) \rightarrow \mathcal{E}_{[\mathcal{M}]}\left(\prod_{i} E_{i}, \prod_{i} F_{i}\right)$.

If $\mathcal{M}$ has also $\left(\mathcal{M}_{[\mathrm{FdB}]}\right)$, then we get

(7) comp : $\mathcal{E}_{[\mathcal{M}]}(F, G) \times \mathcal{E}_{[\mathcal{M}]}(U, F) \rightarrow \mathcal{E}_{[\mathcal{M}]}(U, G)$.

(8) $\mathcal{E}_{[\mathcal{M}]}(\cdot, \cdot): \mathcal{E}_{[\mathcal{M}]}\left(F, F_{1}\right) \times \mathcal{E}_{[\mathcal{M}]}\left(E_{1}, E\right) \rightarrow \mathcal{E}_{[\mathcal{M}]}\left(\mathcal{E}_{[\mathcal{M}]}(E, F), \mathcal{E}_{[\mathcal{M}]}\left(E_{1}, F_{1}\right)\right)$ which is given by $(f, g) \mapsto(h \mapsto f \circ h \circ g)$.

Remark: (7) proves the claim of [9, Remark 4.23.].

\subsection{Comparison of conditions $(\mathbf{m g})$ and $\left(\mathcal{M}_{\{\mathbf{m g}\}}\right)$}

In [6, Example 5.4.] it was shown that cartesian closedness fails for $\mathcal{M}=\{M\}$ if $M$ does not satisfy $(\mathrm{mg})$. In the weight matrix case we can prove the following (counter)-example: 
Example 6.5. There exist (non-constant) $\left(\mathcal{M}_{\mathrm{sc}}\right)$ weight matrices $\mathcal{M}$ with $\left(\mathcal{M}_{\{\mathrm{mg}\}}\right)$ but such that no $M^{x} \in \mathcal{M}$ satisfies (mg)

Proof. Let $\mathcal{M}=\Omega$ be coming from $\omega \in \mathcal{W}$ such that $\left(\omega_{6}\right)$ does not hold, see [9, 5.5., Corollary 5.8. (2)]. The weights $\omega(t):=\max \left\{0, \log (t)^{s}\right\}, s>1$, are concrete examples, see also [2] for the consequences of $\left(\omega_{6}\right)$.

In the next step we generalize [6, Example 5.4.]. We show that $\left(\mathcal{M}_{\{\mathrm{mg}\}}\right)$ is necessary for Theorem 6.2 .

Lemma 6.6. Let $\mathcal{M}$ be $\left(\mathcal{M}_{\mathrm{sc}}\right)$ with $\Lambda=\mathbb{N}_{>0}$ but such that $\left(\mathcal{M}_{\{\mathrm{mg}\}}\right)$ does not hold. Then there exists $f \in \mathcal{E}_{\{\mathcal{M}\}}\left(\mathbb{R}^{2}, \mathbb{C}\right)$ such that the associated mapping $f^{\vee}: \mathbb{R} \rightarrow \mathcal{E}_{\{\mathcal{M}\}}(\mathbb{R}, \mathbb{C})$ is not $\mathcal{E}_{\{\mathcal{M}\}}$.

Proof. We follow the proof of [6, Example 5.4.]. The negation of $\left(\mathcal{M}_{\{\mathrm{mg}\}}\right)$ gives

$$
\exists x \in \Lambda \forall C>0 \forall y \in \Lambda \exists j, k \in \mathbb{N}: M_{j+k}^{x}>C^{j+k} M_{j}^{y} M_{k}^{y} .
$$

For this $x \in \Lambda$ and the choice $C=y=n, n \in \mathbb{N}_{>0}$, we obtain sequences $\left(j_{n}\right)_{n}$ and $\left(k_{n}\right)_{n}$ such that $\left(j_{n}\right)_{n}$ is increasing, $j_{n} \rightarrow \infty, k_{n} \geq 1$ for each $n \in \mathbb{N}_{>0}$ and with

$$
\left(\frac{M_{j_{n}+k_{n}}^{x}}{M_{j_{n}}^{n} M_{k_{n}}^{n}}\right)^{1 /\left(k_{n}+j_{n}\right)} \geq n .
$$

Define a linear functional $\alpha: \mathcal{E}_{\{\mathcal{M}\}}(\mathbb{R}, \mathbb{C}) \rightarrow \mathbb{C}$ by

$$
\alpha(f):=\sum_{n \geq 1}(\sqrt{-1})^{3 j_{n}} \frac{f^{\left(j_{n}\right)}(0)}{M_{j_{n}}^{n} n^{j_{n}}} .
$$

Claim. $\alpha$ is bounded. For given $f \in \mathcal{E}_{\{\mathcal{M}\}}(\mathbb{R}, \mathbb{C})$ we choose $h>0$ and $l \in \Lambda$ large enough and estimate

$$
\begin{aligned}
\left|\sum_{n \geq 0}(\sqrt{-1})^{3 j_{n}} \frac{f^{\left(j_{n}\right)}(0)}{M_{j_{n}}^{n} n^{j_{n}}}\right| \leq \sum_{n \geq 0} \frac{\left|f^{\left(j_{n}\right)}(0)\right|}{h^{j_{n}} M_{j_{n}}^{l}} \frac{M_{j_{n}}^{l}}{M_{j_{n}}^{n}}\left(\frac{h}{n}\right)^{j_{n}} \leq \\
\\
\|f\|_{M^{l},[-1,1], h} \sum_{n \geq 0} \frac{M_{j_{n}}^{l}}{M_{j_{n}}^{n}}\left(\frac{h}{n}\right)^{j_{n}}<+\infty .
\end{aligned}
$$

Note that $M^{l} \leq M^{n}$ for $l \leq n$ and $\sum_{n \geq 0}\left(\frac{h}{n}\right)^{j_{n}}<+\infty$ for each $h>0$.

We apply $\alpha$ to $\tilde{\theta}_{x} \in \mathcal{E}_{\left\{M^{x}\right\}}^{\text {global }}(\mathbb{R}, \mathbb{C})$ (see (2.4)), where $x \in \Lambda$ is the index from (6.3).

For $s, t \in \mathbb{R}$ define $\psi_{x}(s, t):=\tilde{\theta}_{x}(s+t)$ and so $\psi_{x} \in \mathcal{E}_{\{\mathcal{M}\}}^{\text {global }}\left(\mathbb{R}^{2}, \mathbb{C}\right)$ with $\psi_{x}^{\left(\beta_{1}, \beta_{2}\right)}(0,0)=(\sqrt{-1})^{\beta_{1}+\beta_{2}} s_{\beta_{1}+\beta_{2}}^{x}$ for all $\left(\beta_{1}, \beta_{2}\right) \in \mathbb{N}^{2}$. 
Claim. $\alpha \circ \psi_{x}^{\vee}$ is not $\mathcal{E}_{\{\mathcal{M}\}}$. Let $h>0$ and $l \in \Lambda$ be arbitrary (large) but fixed and estimate as follows:

$$
\begin{aligned}
& \left\|\alpha \circ \psi_{x}^{\vee}\right\|_{M^{l},[-1,1], h}=\sup _{t \in[-1,1], k \in \mathbb{N}} \frac{\left|\left(\alpha \circ \psi_{x}^{\vee}\right)^{(k)}(t)\right|}{h^{k} M_{k}^{l}} \\
& \geq \sup _{k \in \mathbb{N}} \frac{1}{h^{k} M_{k}^{l}}\left|\sum_{n \geq 1}(\sqrt{-1})^{3 j_{n}} \frac{\psi_{x}^{\left(j_{n}, k\right)}(0,0)}{M_{j_{n}}^{n} n^{j_{n}}}\right| \\
& =\sup _{k \in \mathbb{N}} \frac{1}{h^{k} M_{k}^{l}}\left|\sum_{n \geq 1}(\sqrt{-1})^{3 j_{n}} \frac{(\sqrt{-1})^{j_{n}+k} s_{j_{n}+k}^{x}}{M_{j_{n}}^{n} n^{j_{n}}}\right|=\sup _{k \in \mathbb{N}} \frac{1}{h^{k} M_{k}^{l}}\left|(\sqrt{-1})^{k} \sum_{n \geq 1} \frac{s_{j_{n}+k}^{x}}{M_{j_{n}}^{n} n^{j_{n}}}\right| \\
& =\sup _{k \in \mathbb{N}} \frac{1}{h^{k} M_{k}^{l}} \sum_{n \geq 1} \frac{s_{j_{n}+k}^{x}}{M_{j_{n}}^{n} n^{j_{n}}} \underbrace{\geq}_{k=k_{n}} \sup _{n \in \mathbb{N}_{>0}} \frac{1}{h^{k_{n}} M_{k_{n}}^{l}} \frac{M_{k_{n}}^{n}}{M_{k_{n}}^{n}} \frac{s_{j_{n}+k_{n}}^{x}}{M_{j_{n}}^{n} n^{j_{n}}} \\
& \geq \sup _{n \in \mathbb{N}_{>0}} \frac{M_{k_{n}}^{n}}{h^{k_{n}} n^{j_{n}} M_{k_{n}}^{l}} \frac{M_{j_{n}+k_{n}}^{x}}{M_{j_{n}}^{n} M_{k_{n}}^{n}} \geq \sup _{n \in \mathbb{N}_{>0}} \frac{n^{j_{n}+k_{n}}}{h^{k_{n}} n^{j_{n}}} \frac{M_{k_{n}}^{n}}{M_{k_{n}}^{l}}=+\infty .
\end{aligned}
$$

\section{Remarks and special cases}

\subsection{More results for $\mathcal{E}_{[\mathcal{M}]}$}

Let $\mathcal{M}$ be $(\mathcal{M})$ with $\Lambda=\mathbb{R}_{>0}$. Using the closed graph theorem [4, 52.10] the matrix generalization of the uniform boundedness principle [6, Theorem 6.1.] is valid for $\mathcal{E}_{[\mathcal{M}]}$, see [12, Theorem 12.4.1.]. All further results from [6, Chapter 8] can be transferred to the matrix-case, see [12, 12.4., 12.6., 12.7.]. For the generalization of [6, Theorem 2.2.] see [12, Proposition 9.4.4.].

Let $\mathcal{M}$ be $(\mathcal{M})$ and assume that

(i) $\mathcal{M}$ is $\left(\mathcal{M}_{\mathrm{sc}}\right)$ with $\Lambda=\mathbb{R}_{>0}$ and has

(ii) $\left(\mathcal{M}_{[\mathrm{mg}]}\right)\left(\Rightarrow\left(\mathcal{M}_{[\mathrm{dc}]}\right)\right)$;

(iii) for the Roumieu-case $\left(\mathcal{M}_{\mathcal{H}}\right)$, for the Beurling-case $\left(\mathcal{M}_{\left(\mathcal{C}^{\omega}\right)}\right)$;

(iv) $\left(\mathcal{M}_{[\mathrm{FdB}]}\right)$ or equivalently $\left(\mathcal{M}_{[\text {rai] }}\right)$ (see [10, Lemma 1]).

Using [10, Theorems 5,6], where we characterized the required stability properties for $\mathcal{E}_{[\mathcal{M}]}$, all results from [6, Chapter 9] can be transferred to the $\mathcal{E}_{[\mathcal{M}]}$-case, see [12, 12.8.] for full proofs. Note that the characterization theorem for the Beurlingcase shown in [12, Chapter 8] is weaker than [10, Theorem 6]. 


\subsection{Special cases $\mathcal{M}=\{M\}$ and $\mathcal{M}=\Omega$}

To apply all previous results to the constant case $\mathcal{M}=\{M\}$ we have to assume that

(i) $M \in \mathcal{L C}$;

(ii) $\liminf _{p \rightarrow \infty}\left(m_{p}\right)^{1 / p}>0$ in the Roumieu-, $\lim _{p \rightarrow \infty}\left(m_{p}\right)^{1 / p}=+\infty$ in the Beurling-case;

(iii) $M$ has $(\mathrm{mg})(\Rightarrow(\mathrm{dc})$,

(iv) $M$ has (FdB) or equivalently (rai) (see also [9, Chapter 3]).

If $\mathcal{M}=\Omega=\left\{\left(\Omega_{j}^{l}\right)_{j}: l>0\right\}$ with $\Omega_{j}^{l}:=\exp \left(1 / l \varphi_{\omega}^{*}(l j)\right)$, then we assume that $\omega \in \mathcal{W}$ and

(i) $\left(\omega_{2}\right)$ in the Roumieu-, $\left(\omega_{5}\right)$ in the Beurling-case to guarantee $\left(\mathcal{M}_{\mathcal{H}}\right)$ respectively $\left(\mathcal{M}_{\left(\mathcal{C}^{\omega}\right)}\right)($ see $[9$, Corollary 5.15.]);

(ii) $\left(\omega_{1^{\prime}}\right)$, i.e. $\omega$ is equivalent w.r.t. $\sim$ to a sub-additive weight, see [10, Theorems 3,4] and [9, Chapter 6].

\subsection{Weight matrices in the sense of Beaugendre, Schmets and Valdivia}

Beaugendre in [1] and Schmets and Valdivia in [13] have considered weight matrices in the following sense: Let $\Phi:[0,+\infty) \rightarrow \mathbb{R}$ be a convex and increasing function with $\lim _{t \rightarrow \infty} \frac{\Phi(t)}{t}=+\infty$ and $\Phi(0)=0$ (w.l.o.g. - replace $\Phi$ by $\Psi(t):=\Phi(t)-\Phi(0)$, see [13, Definition 16.]). We introduce the following weight matrix

$$
\mathcal{M}^{\Phi}:=\left\{\left(p ! m_{a p}^{\Phi}\right)_{p \in \mathbb{N}}: a>0\right\} \quad m_{a p}^{\Phi}:=\exp (\Phi(a p)) .
$$

In the literature the Beurling-case $\mathcal{E}_{\left(\mathcal{M}^{\Phi}\right)}$ was considered. We summarize some properties:

(i) $\mathcal{M}^{\Phi}$ is $\left(\mathcal{M}_{\mathrm{sc}}\right)$ and $\left(\mathcal{M}_{\left(\mathcal{C}^{\omega}\right)}\right)$ holds.

(ii) $\left(\mathcal{M}_{\{\mathrm{L}\}}\right)$ and $\left(\mathcal{M}_{(\mathrm{L})}\right)$ both are satisfied, compare this with [9, Lemma 5.9. (5.10)] where condition $\left(\omega_{1}\right)$ is needed. As shown in [13, Lemma 17] we get both

$$
\begin{aligned}
\forall a>0 \forall h>0 \exists b>0(b>a) & \exists D>0 \forall p \in \mathbb{N}_{>0}: \\
& \log (h)-\frac{\log (D)}{p} \leq \frac{1}{p}(\Phi(b p)-\Phi(a p))
\end{aligned}
$$

and

$$
\begin{aligned}
\forall b>0 \forall h>0 \exists a>0(a<b) & \exists D>0 \forall p \in \mathbb{N}_{>0}: \\
& \log (h)-\frac{\log (D)}{p} \leq \frac{1}{p}(\Phi(b p)-\Phi(a p)),
\end{aligned}
$$


since convexity of $\Phi$ yields

$$
\begin{aligned}
\forall a, b>0, b>a: \frac{\Phi(b p)-\Phi(a p)}{p(b-a)} \geq \frac{\Phi(b p)}{p b} & \Leftrightarrow \\
\frac{\Phi(b p)-\Phi(a p)}{p} & \geq \frac{\Phi(b p)}{p b}(b-a) \rightarrow \infty
\end{aligned}
$$

as $p \rightarrow \infty$.

(iii) (7.1) implies also that all sequences are pairwise not equivalent. If $\left(m_{a p}^{\Phi}\right)_{p} \approx\left(m_{b p}^{\Phi}\right)_{p}$ for all $a, b>0$, then we would get

$$
\begin{aligned}
\forall a>0 \forall b>0 \exists C \geq 1 \forall p \in \mathbb{N}: \quad m_{b p}^{\Phi} \leq C^{p} m_{a p}^{\Phi} \Leftrightarrow \\
\quad \frac{1}{p}(\Phi(b p)-\Phi(a p)) \leq \log (C),
\end{aligned}
$$

but the left hand side tends to infinity as $p \rightarrow \infty$ whenever $b>a$. So $\mathcal{M}^{\Phi}$ has both $\left(\mathcal{M}_{\text {\{strict }}\right)$ and $\left(\mathcal{M}_{\text {(strict })}\right)$.

(iv) $\mathcal{M}^{\Phi}$ has $\left(\mathcal{M}_{\{\mathrm{mg}\}}\right)$ and $\left(\mathcal{M}_{(\mathrm{mg})}\right)$. By convexity of $\Phi$ we get $\Phi(a p+a q) \leq$ $\frac{1}{2} \Phi(2 a p)+\frac{1}{2} \Phi(2 a q) \leq \Phi(2 a p)+\Phi(2 a q)$ for all $a>0$ and $p, q \in \mathbb{N}$ and so

$$
M_{a(p+q)}^{\Phi} \leq M_{b p}^{\Phi} \cdot M_{b q}^{\Phi} \Leftrightarrow \Phi(a(p+q)) \leq \Phi(b p)+\Phi(b q)
$$

holds with $b=2 a$.

(v) $\left(\mathcal{M}_{\{\mathrm{FdB}\}}\right)$ and $\left(\mathcal{M}_{(\mathrm{FdB})}\right)$ both are satisfied. This is clear since each $\left(m_{a p}^{\Phi}\right)_{p}$ is log-convex, see e.g. [9, 2.2. Lemma (1)].

Thus also for $\mathcal{E}_{\left[\mathcal{M}^{\Phi]}\right.}$ the exponential laws in Theorem 6.2 and the consequences in Lemma 6.3 are valid. Moreover the characterizing results [10, Theorems 5,6] and all further generalizations of the results from [6] hold.

As special case one may consider $\Phi=\varphi_{\omega}^{*}$ for $\omega \in \mathcal{W}$. Then on the one hand one has the matrix $\mathcal{M}^{\Phi}$ as defined before, on the other hand the weight matrix $\Omega:=\left\{\left(\Omega_{p}^{a}\right)_{p}:=\exp \left(1 / a \varphi_{\omega}^{*}(a p)\right): a>0\right\}$ as the approach in [3]. By definition we have

$$
m_{a p}^{\Phi}:=\exp (\Phi(a p))=\exp \left(1 / a \varphi_{\omega}^{*}(a p)\right)^{a}=\left(\Omega_{p}^{a}\right)^{a} .
$$

As we have already pointed out the weights $\omega_{s}:=\max \left\{0, \log (t)^{s}\right\}, s>1$, generate an infinite non-constant weight matrix. We denote the associated matrices by $\mathcal{M}_{s}^{\Phi}$ and $\Omega_{S}$ and prove:

Lemma 7.4. For any $s>1$ the matrices $\mathcal{M}_{s}^{\Phi}$ and $\Omega_{s}$ are equivalent w.r.t. both $\{\approx\}$ and $(\approx)$,

Proof. Let $s>1$ be arbitrary but fixed. For $t \geq 0$ we get $\varphi_{\omega_{s}}(t)=\omega_{s}(\exp (t))=$ $(\log (\exp (t)))^{s}=t^{s}$, hence $\varphi_{\omega_{s}}^{*}(x)=\sup \left\{x y-y^{s}: y \geq 0\right\}=: \sup \left\{f_{x, s}(y): y \geq 0\right\}$ for all $x \geq 0$. A straightforward computation shows

$$
\varphi_{\omega_{s}}^{*}(x)=f_{x, s}\left(\left(\frac{x}{s}\right)^{\frac{1}{s-1}}\right)=x\left(\frac{x}{s}\right)^{\frac{1}{s-1}}-\left(\frac{x}{s}\right)^{\frac{s}{s-1}}=x^{\frac{s}{s-1}}\left(\frac{1}{s^{\frac{1}{s-1}}}-\frac{1}{s^{\frac{s}{s-1}}}\right)=: x^{\frac{s}{s-1}} R(s)
$$


and so

$$
\Omega_{p}^{l}=\exp \left(l^{1 /(s-1)} p^{s /(s-1)} R(s)\right) \quad m_{l p}^{\Phi}=\exp \left(l^{s /(s-1)} p^{s /(s-1)} R(s)\right) .
$$

The case $s=2$ gives $\Omega_{p}^{l}=(\exp (l R(2)))^{p^{2}}=(\exp (l / 4))^{p^{2}}$ and $m_{l p}^{\Phi}=\left(\exp \left(l^{2} / 4\right)\right)^{p^{2}}$.

$\Omega_{\S\{\preceq\}} \mathcal{M}_{s}^{\Phi}$. Let $l \in \mathbb{N}_{>0}$ (large) and get $\Omega_{p}^{l} \leq\left(\Omega_{p}^{l}\right)^{l} \leq\left(\Omega_{p}^{l}\right)^{l} p !=p ! m_{l p}^{\Phi}$ for each $p \in \mathbb{N}$ since $\Omega_{p}^{l} \geq 1$ for each $l>0, p \in \mathbb{N}$.

$\mathcal{M}_{s}^{\Phi}\{\preceq\} \Omega_{s}$. Let $l>0$, then we have to find $n>l>0$ and $C \geq 1$ such that for all $p \in \mathbb{N}$ we get $p ! m_{l p}^{\Phi} \leq C^{p} \Omega_{p}^{n} \Leftrightarrow p ! \exp \left(l^{s /(s-1)} p^{s /(s-1)} R(s)\right) \leq$ $C^{p} \exp \left(n^{1 /(s-1)} p^{s /(s-1)} R(s)\right)$. So the choice $n=2^{s-1} l^{s}$ is sufficient and analogously $\mathcal{M}_{s}^{\Phi}(\preceq) \Omega_{s}$ holds, too.

$\Omega_{\S}(\preceq) M_{s}^{\Phi}$. For each $l>0$ (small) there exists $C \geq 1$ and $n>0$ such that for all $p \in \mathbb{N}$ we get

$$
\Omega_{p}^{n} \leq C^{p} p ! m_{l p}^{\Phi} \Leftrightarrow \exp \left(n^{1 /(s-1)} p^{s /(s-1)} R(s)\right) \leq C^{p} p ! \exp \left(l^{s /(s-1)} p^{s /(s-1)} R(s)\right),
$$

so the choice $n=l^{s}$ is sufficient.

If $\omega \in \mathcal{W}$, then $\Omega$ has always both $\left(\mathcal{M}_{\{\mathrm{mg}\}}\right)$ and $\left(\mathcal{M}_{(\mathrm{mg})}\right)$. But $\Omega^{l} \approx \Omega^{n}$ for all $l, n>0$ holds if and only if $(\mathrm{mg})$ for some/each $\Omega^{l}$ and if and only if $\left(\omega_{6}\right)$ for $\omega$, see [9, Chapter 5].

For $\mathcal{M}^{\Phi}$ this is not true any more. As we have already seen the sequences in $\mathcal{M}^{\Phi}$ are always pairwise not equivalent.

On the other hand, since $\left(m_{a p}^{\Phi}\right)_{p \in \mathbb{N}}$ is log-convex, (mg) holds for this sequence if and only if $m_{a 2 p}^{\Phi} \leq C^{2 p}\left(m_{a p}^{\Phi}\right)^{2} \Leftrightarrow \frac{1}{2 p} \Phi(2 a p)-\frac{1}{p} \Phi(a p) \leq \log (C)$ for a constant $C \geq 1$ and all $p \in \mathbb{N}$, see [8, Theorem 1, (3) $\Rightarrow(2)]$. So if $\Phi$ satisfies

$$
\exists D \geq 1 \forall t \geq 0: \Phi(2 t) \leq 2 \Phi(t)+D t,
$$

then each $\left(m_{a p}^{\Phi}\right)_{p \in \mathbb{N}}$ has (mg), In [1] a weight with (7.3) is called a weight of moderate growth. (7.3) is valid for $\Phi=\varphi_{\omega}^{*}$ if and only if $\omega \in \mathcal{W}$ has $\left(\omega_{6}\right)$. This holds by the proof of (5.11.) in [9. Lemma 5.9.] and by applying the conjugate operator to (7.3) (note that $\varphi_{\omega}^{* *}=\varphi_{\omega}$ ).

Finally consider $\Phi(t):=t \log (t)$ for $t \geq 1$ and $\Phi(t):=0$ for $0 \leq t<1$. Each $\left(m_{a p}^{\Phi}\right)_{p \in \mathbb{N}}$ has (mg), since $\frac{1}{2 p} \Phi(2 a p)-\frac{1}{p} \Phi(a p)=a \log (2)$. More precisely Stirling's formula and $m_{a p}^{\Phi}=\exp (\Phi(a p))=(a p)^{a p}$ show that this yields the Gevreymatrix $\mathcal{G}$ and which should be compared with [9, 5.19.]. 


\section{References}

[1] P. Beaugendre. Extensions de jets dans des intersections de classes non quasi-analytiques. Ann. Polon. Math., 76(3):213-243, 2001.

[2] J. Bonet, R. Meise, and S. N. Melikhov. A comparison of two different ways to define classes of ultradifferentiable functions. Bull. Belg. Math. Soc. Simon Stevin, 14:424-444, 2007.

[3] R. W. Braun, R. Meise, and B. A. Taylor. Ultradifferentiable functions and Fourier analysis. Results Math., 17(3-4):206-237, 1990.

[4] A. Kriegl and P. W. Michor. The convenient setting of global analysis, volume 53 of Mathematical Surveys and Monographs. American Mathematical Society, Providence, RI, 1997. http://www . ams .org/online_bks/surv53/.

[5] A. Kriegl, P. W. Michor, and A. Rainer. The convenient setting for nonquasianalytic Denjoy-Carleman differentiable mappings. J. Funct. Anal., 256:3510-3544, 2009.

[6] A. Kriegl, P. W. Michor, and A. Rainer. The convenient setting for Denjoy-Carleman differentiable mappings of Beurling and Roumieu type, 2011. accepted for publication in Rev. Mat. Complut., available online at http://arxiv.org/pdf/1111.1819.pdf.

[7] A. Kriegl, P. W. Michor, and A. Rainer. The convenient setting for quasianalytic Denjoy-Carleman differentiable mappings. J. Funct. Anal., 261(7), 2011.

[8] W. Matsumoto. Characterization of the separativity of ultradifferentiable classes. J. Math. Kyoto Univ., 24(4):667-678, 1984.

[9] A. Rainer and G. Schindl. Composition in ultradifferentiable classes. Studia Mathematica, 224(2):97-131, 2014.

[10] A. Rainer and G. Schindl. Equivalence of stability properties for ultradifferentiable function classes, 2014. accepted for publication in Rev. R. Acad. Cienc. Exactas Fis. Nat. Ser. A Math. RACSAM, available online at http://arxiv.org/pdf/1407.6673.pdf.

[11] G. Schindl. Spaces of smooth functions of DenjoyCarleman-type, 2009. Diploma Thesis, available online at http://othes.univie.ac.at/7715/1/2009-11-18_0304518.pdf.

[12] G. Schindl. Exponential laws for classes of Denjoy-Carlemandifferentiable mappings, 2014. PhD Thesis, available online at http://othes.univie.ac.at/32755/1/2014-01-26_0304518.pdf. 
[13] J. Schmets and M. Valdivia. Extension properties in intersections of non quasi-analytic classes. Note di Matematica, 25(2):159-185, 2006.

[14] V. Thilliez. On quasi-analytic local rings. Expo. Math., 26:1-23, 2008.

Fakultät für Mathematik, Universität Wien, Oskar-Morgenstern-Platz 1, A-1090 Wien, Austria email: a0304518@unet.univie.ac.at 